\title{
A CCR4 antagonist reverses the tumor-promoting microenvironment of renal cancer
}

\author{
Chiara Berlato, ${ }^{1}$ Moddasar N. Khan, ${ }^{1}$ Tiziana Schioppa,, ${ }^{1,2}$ Richard Thompson, ${ }^{1}$ Eleni Maniati, ${ }^{1}$ Anne Montfort, ${ }^{1}$ Maryam Jangani, ${ }^{1}$ \\ Monica Canosa, ${ }^{1}$ Hagen Kulbe, ${ }^{1,3}$ Urs B. Hagemann, ${ }^{4}$ Alexander R. Duncan, ${ }^{4}$ Laura Fletcher, ${ }^{5}$ Robert W. Wilkinson, ${ }^{6}$ \\ Thomas Powles, ${ }^{1}$ Sergio A. Quezada, ${ }^{7}$ and Frances R. Balkwill ${ }^{1}$ \\ 'Barts Cancer Institute, Queen Mary University of London, London, United Kingdom. '2Department of Molecular and Translational Medicine, University of Brescia, Brescia, Italy. ${ }^{3}$ Department of Gynecology, \\ Charité Universitätsmedizin, Berlin, Germany. . Affitech Research AS, 0slo, Norway. ${ }^{5}$ Cancer Research Technology Ltd., London, United Kingdom. ${ }^{6}$ Medlmmune Ltd., Cambridge, United Kingdom. ${ }^{7}$ Cancer \\ Immunology Unit, Research Department of Haematology, UCL Cancer Institute, University College London, London, United Kingdom.
}

\begin{abstract}
Elevated expression of the chemokine receptor CCR4 in tumors is associated with poor prognosis in several cancers. Here, we have determined that CCR4 was highly expressed in human renal cell carcinoma (RCC) biopsies and observed abnormal levels of CCR4 ligands in RCC patient plasma. An antagonistic anti-CCR4 antibody had antitumor activity in the RENCA mouse model of RCC. CCR4 inhibition did not reduce the proportion of infiltrating leukocytes in the tumor microenvironment but altered the phenotype of myeloid cells, increased NK cell and Th1 cytokine levels, and reduced immature myeloid cell infiltrate and blood chemokine levels. In spite of prominent changes in the myeloid compartment, the anti-CCR4 antibody did not affect RENCA tumors in T cell-deficient mice, and treatment with an anti-class II MHC antibody abrogated its antitumor activity. We concluded that the effects of the anti-CCR4 antibody required the adaptive immune system and CD4+ $T$ cells. Moreover, CCL17-induced IFN- $\gamma$ production was reduced when Th1-polarized normal CD4+ $\mathrm{T}$ cells were exposed to the CCR4 ligand, evidencing the involvement of CCR4 in Th1/Th2 regulation. The anti-CCR4 antibody, alone or in combination with other immune modulators, is a potential treatment approach to human solid cancers with high levels of CCR4-expressing tumor-infiltrating leukocytes and abnormal plasma CCR4 ligand levels.
\end{abstract}

\section{Introduction}

Tumor microenvironments possess complex chemokine networks that contribute to the extent and phenotype of the host infiltrate (1-3). In addition, malignant cells may gain functional chemokine receptors, often as a consequence of oncogenic mutations, allowing them to respond to distant chemokine gradients during metastatic spread $(4,5)$.

The chemokine receptor CCR4 is expressed on circulating and tissue-resident $\mathrm{T}$ cells, being predominantly associated with a Th2 phenotype (6-8), as well as on other T helper cells (9). CCR4 is also highly expressed on circulating Tregs and on Tregs recruited at tumor sites in ovarian cancer (10) and in glioblastoma (11). In ovarian cancer, the CCR4 ligand CCL22 is found both in the tumor tissue and in macrophages isolated from ascitic fluid (9). In hepatocellular carcinoma, malignant cell-produced CCL22 recruited $\mathrm{CCR}^{+}$Tregs that facilitated immune escape of malignant cells (12). Similarly, in breast cancer, CCR $4^{+}$Tregs, recruited by CCL22 in the tumor microenvironment, are predictive of a worse progno-

Conflict of interest: Some of the research in this paper was funded by Affitech Research AS as well as the salary for C. Berlato for twenty-one months. C. Berlato, M.N. Khan, T. Schioppa, and F.R. Balkwill are inventors on the patent CCR4 Cancer Marker and Therapeutic Target Patent (PCT/CB2008/003160).

License: This work is licensed under the Creative Commons Attribution 4.0 International License. To view a copy of this license, visit http://creativecommons.org/ licenses/by/4.0/

Submitted: June 8, 2015; Accepted: December 1, 2016.

Reference information: / Clin Invest. 2017;127(3):801-813.

https://doi.org/10.1172/JCl82976. sis (13). A second breast cancer study found reduced overall survival and high CCR4 expression in tumor biopsies (14). Finally, in a cohort of 753 patients with gastric adenocarcinoma, positive staining for CCR4 was also associated with a poorer prognosis (15).

CCR4 also plays a role in hematological malignancies, and there are now clinical trials of an anti-CCR4 antibody, mogamulizumab, that has enhanced antibody-dependent cell-mediated cytotoxicity (ADCC) activity. Mogamulizumab is approved in Japan for the treatment of relapsed adult T cell leukemia (ATL) (16) and has also been tested in patients with relapsed peripheral T cell lymphoma (PTLC) and cutaneous T cell lymphoma (CTLC) (17). The treatment is indicated for patients with CCR4-positive leukemia cells, but might also act by reducing the number of Tregs in cancer patients (18).

In this article, we have investigated CCR4 as a target in renal cell carcinoma (RCC) using patient samples and an orthotopic mouse RCC model. We have found abnormal levels of CCR4 and its ligands in human RCC biopsies and plasma samples. In preclinical experiments we found that Affi-5, a fully human antiCCR4 antibody with antagonistic activity (described in ref. 19), has antitumor activity in a renal cancer model. Inhibition of CCR4 did not reduce the proportion of CCR4-positive infiltrating leukocytes in the tumor microenvironment but altered the phenotype of the immune infiltrate, affecting in particular the phenotype of myeloid cells and increasing the number of infiltrating NK cells. These effects were dependent on the adaptive immune system and required functioning $\mathrm{CD} 4^{+} \mathrm{T}$ cells. The antibody also altered the phenotype of tumor-associated macrophages (TAMs) in the B16 melanoma model. Inhibition of CCR4, alone or in combina- 
A

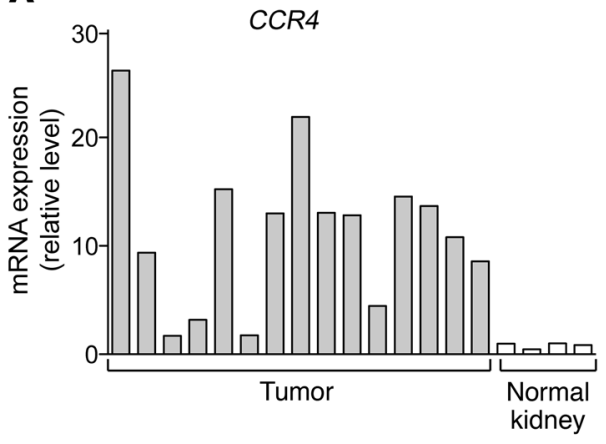

C

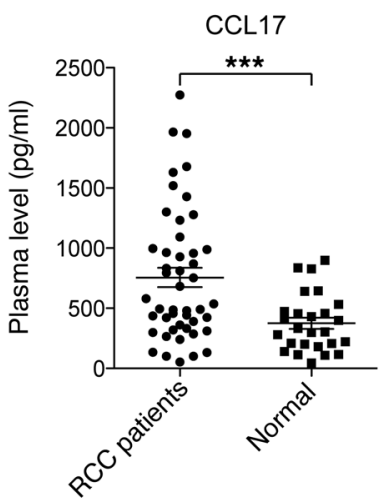

B
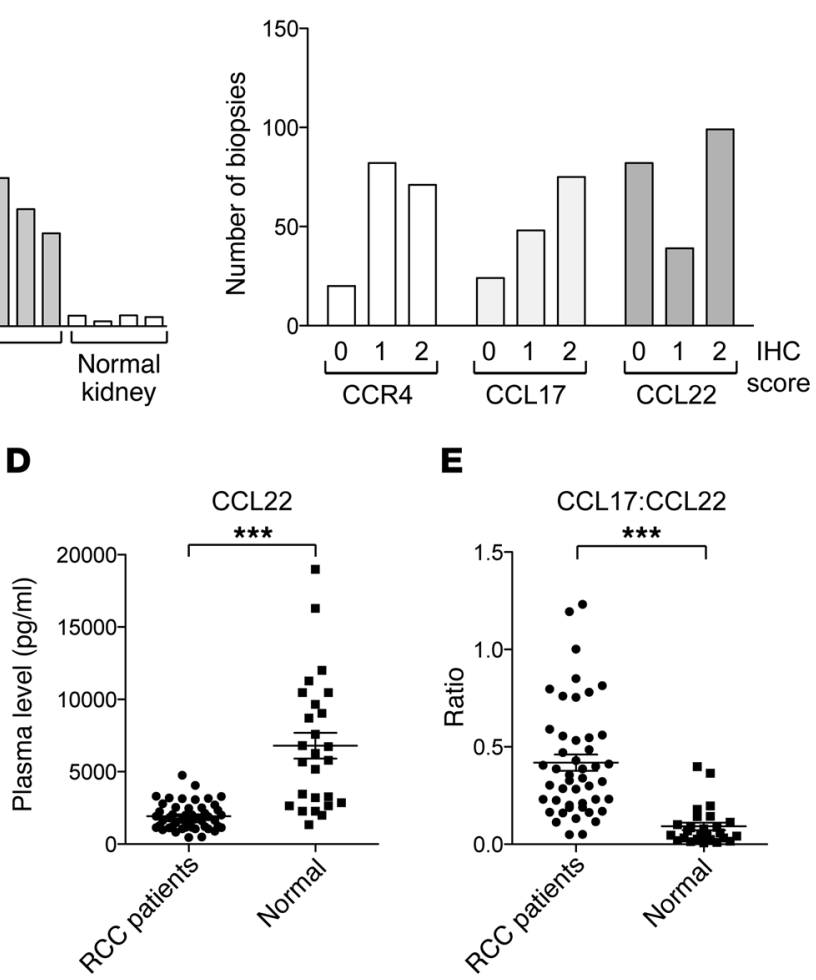

E

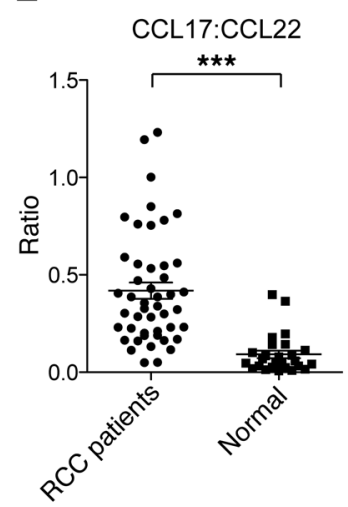

Figure 1. Abnormal expression of CCR4 and its ligands in human renal cancer. (A) CCR4 mRNA was measured by real-time RT-PCR in RCC biopsies and compared with normal kidney. (B) Levels of CCR4 and its ligands CCL17 and CCL22 were analyzed by IHC in a TMA of renal cancer biopsies from human patients. Each biopsy was scored 0 , no staining; 1 , weak staining; 2 , strong staining for CCR4, CCL17, and CCL22. A total of 173 biopsy cores from 57 patients were stained for CCR4 and CCL22, and 145 cores from 48 patients for CCL17. (C-E) Plasma levels of CCL17 and CCL22 and the CCL17/CCL22 ratio in RCC patient plasma were compared with those from normal individuals of matched age using Meso Scale Discovery System Ultra-Sensitive plates. $n=47$ for RCC patients, $n=26$ for normal individuals; 2-tailed Student's $t$ test, ${ }^{* *} P=0.0001$ for CCL17 (C), CCL22 (D), and CCL17/CCL22 (E). (F and G) Kaplan-Meier survival curves for progression-free survival (PFS; F) and overall survival (OS; G) for RCC patients with CCL17/CCL22 high (above the median) or low $(n=57)$. For progression-free survival, hazard ratio $0.436,95 \%$ CI 0.239-0.797; for overall survival, hazard ratio $0.552,95 \% \mathrm{Cl} 0.306-0.995$.
$\mathbf{F}$

PFS - plasma CCL17:CCL22

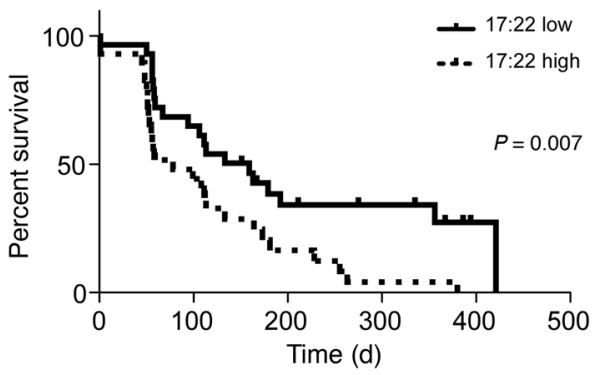

G

OS - plasma CCL17:CCL22

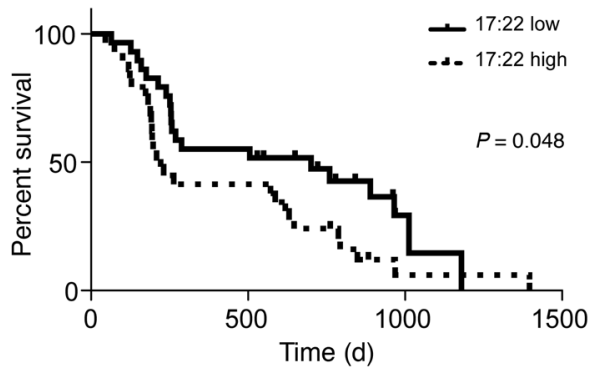

tion with other immune modulators, may be a valuable therapeutic approach in human cancers with high levels of CCR4 in the tumor microenvironment and abnormal plasma CCR4 ligand levels.

\section{Results}

CCR4 and its ligands in human renal cell carcinoma. This study was prompted by the finding of abundant CCR 4 mRNA in biopsies from renal cancers as compared with normal kidney (Figure 1A). CCR4 protein was also detected by IHC on malignant cells and leukocytes in a tissue microarray (TMA) constructed from 57 advanced RCC patient biopsies (Figure 1B and Supplemental Figure 1A; supplemental material available online with this article; https://doi.org/10.1172/JCI82976DS1). Of the 173 cores in the TMA, 157 showed positive CCR4 staining. $75 \%$ of the biopsies were classified as clear cell, with others classified as papillary RCC. There was a significant positive correlation between CCR4 positivity and the extensive T cell $\left(\mathrm{CD}^{+}\right)$or macrophage $\left(\mathrm{CD}^{+} 8^{+}\right)$infiltrates in the tumor cores (Supplemental Figure 1,
B-D), suggesting that CCR4 may be important in the trafficking of tumor-associated leukocytes.

The CCR4 ligands CCL22 (also known as MDC) and CCL17 (also known as TARC) were also expressed in the RCC tumors (Figure 1B and Supplemental Figure 1A). CCR4 was weakly expressed in normal kidney, but the ligands could be detected in normal kidney tubules (Supplemental Figure 1A).

We next compared plasma concentrations of CCL17 and CCL22 from patients with advanced RCC with age-matched controls using the Meso Scale Discovery electrochemiluminescence system. The concentration of CCL17 in plasma was significantly higher in patients; however, the concentration of CCL22 was significantly lower in the patients compared with controls (Figure 1, C and D). The CCL17/CCL22 ratio was also significantly different between the 2 groups and 4 -fold higher in patients compared with controls (Figure 1E). Moreover, a high CCL17/CCL22 ratio correlated with lower progression-free and overall survival rates (Figure 1, F and G), but this association was not seen if the individual chemokines were 
A

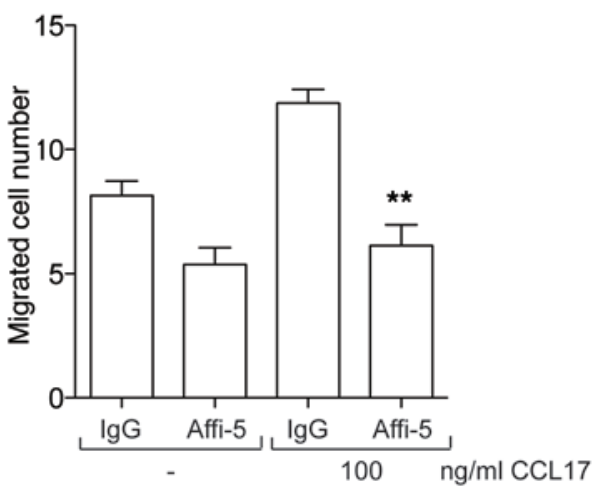

C

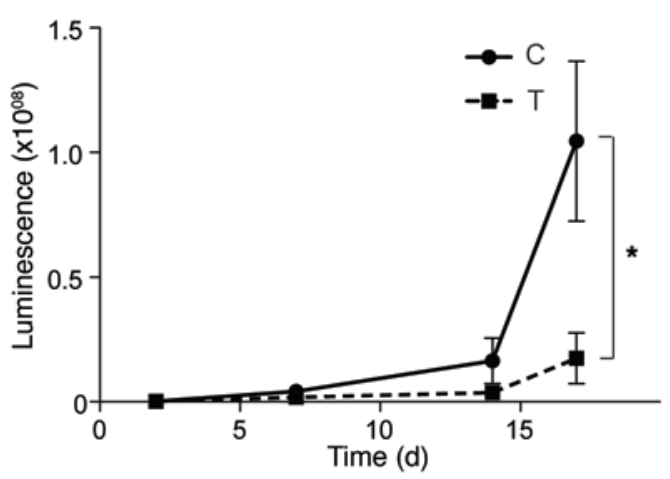

B

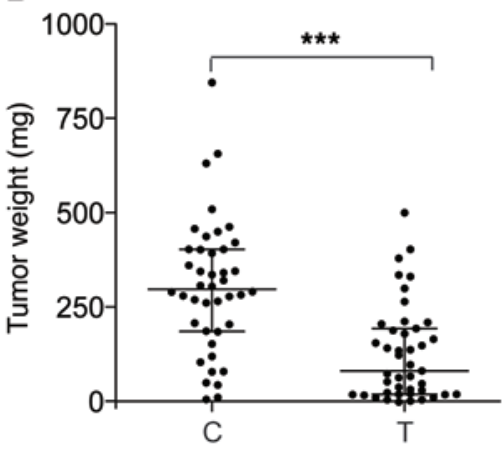

Control

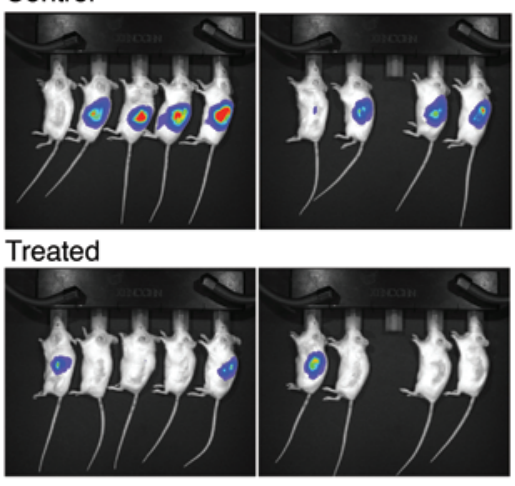

Luminescence

$-60000$

$-40000$

20000
D

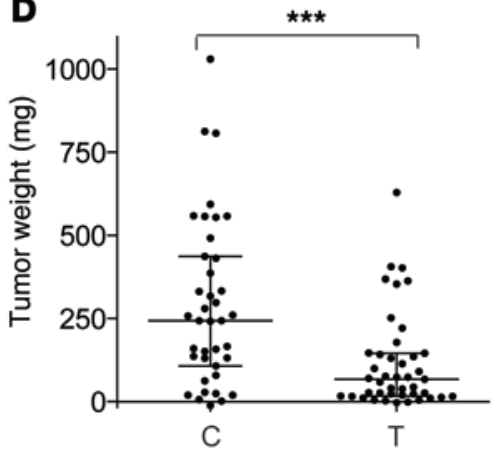

E

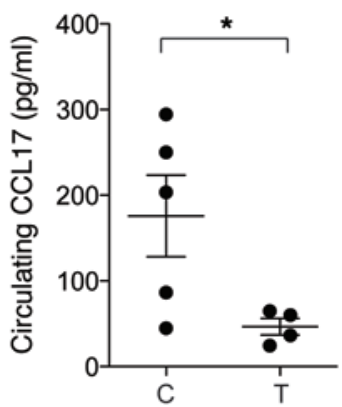

$\mathbf{F}$

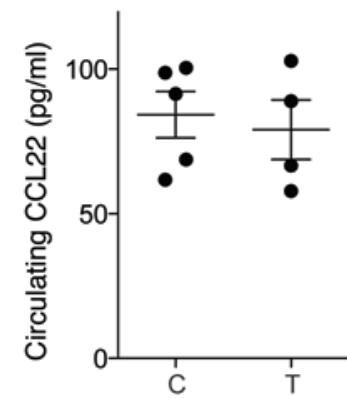

Figure 2. Anti-CCR4 antibody Affi-5 has antitumor activity in the RENCA RCC model. (A) Migration of RENCA cells in response to mouse CCL17 in the presence of $10 \mu \mathrm{g} / \mathrm{ml}$ Affi-5 or isotype control lgG after an incubation of 16 hours was analyzed with a migration assay. Significant difference compared with CCL17-induced migration in the presence of isotype control is indicated; 2-tailed Student's $t$ test, ${ }^{* *} P<0.01$. One experiment representative of 3 is shown. (B and C) BALB/c mice were injected with $1 \times 10^{5}$ RENCA-luc cells and treated twice weekly with Affi- 5 (T) or isotype control (C) at $20 \mathrm{mg} / \mathrm{kg}$ starting 48 hours after surgery. Mice were sacrificed 17 days after surgery, and tumor weight was determined. Combined results of 6 experiments are shown ( $n=39$, $n=43$ T, 2-tailed Student's $t$ test, ${ }^{* * *} P=0.0003$ ). (C) Chemiluminescence was determined on days 7, 14, and 17, and one representative experiment is reported in the graph and images as relative luminescence units. 2-tailed Student's $t$ test, ${ }^{*} P=0.029$ on day 17. (D) BALB/c mice were injected with $1 \times 10^{5}$ RENCA-luc cells and treated twice weekly with Affi- 5 (T) or isotype control (C) at $10 \mathrm{mg} / \mathrm{kg}$ starting 48 hours after surgery. Mice were sacrificed 17 days after surgery, and tumor weight was determined. Combined results of 6 experiments are shown ( $n=39 \mathrm{C}, n=43$ T, 2-tailed Student's $t$ test, $\left.{ }^{* * *} P<0.0001\right)$. (E and F) Serum collected at end point was analyzed by ELISA for CCL17 and CCL22. Two-tailed Student's $t$ test, ${ }^{*} P=0.05, n=5$ for samples from control-treated (C) mice; $n=4$ for samples from Affi-5-treated $(T)$ mice.

examined (Supplemental Figure 1, E and F), suggesting that activity of both chemokines is important in RCC biology. In our cohort, CCR4 expression, as determined by IHC on the TMAs, was not predictive of clinical outcome (data not shown).

Renal cancer cell lines have functional CCR 4 receptors. As we had detected CCR4 and its ligands in malignant cells in tumor biopsies, we next studied RCC cancer cell lines. RCC cell lines 786-O and A498 (human) and RENCA (murine) expressed cell surface CCR4 as determined by flow cytometry. The human cell lines had detectable intracellular CCL17 and CCL22 (Supplemental Figure 2), and these chemokines were also present in the tissue culture medium during 3 days of incubation (CCL17 $300 \mathrm{pg} / 10^{6}$ cells, CCL22 $2 \mathrm{ng} / 10^{6}$ cells). RENCA cells also secreted CCL17 (200 pg/10 6 cells) and CCL22 (10 $\mathrm{pg} / 10^{6}$ cells) in the medium during 3 days of incubation.

Both CCR4 ligands stimulated migration of the human cell line 786-O (Supplemental Figure 3, A and B). Similar data were 
A

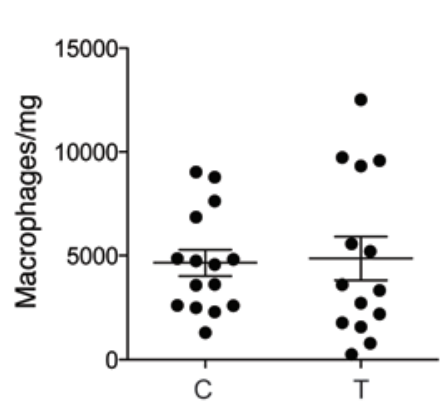

B
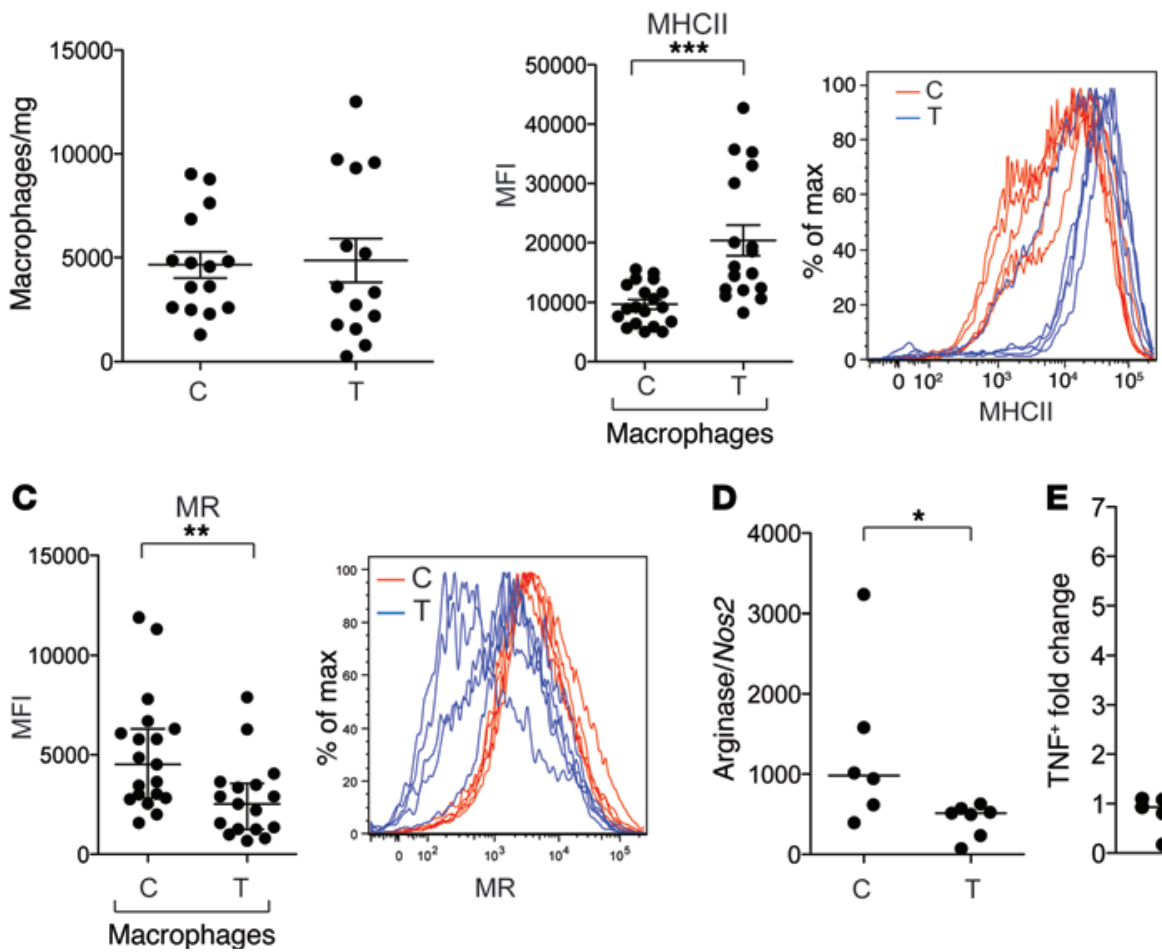

D
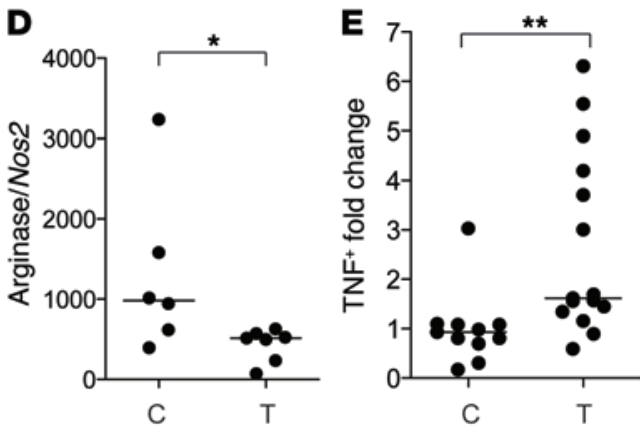

Figure 3. Effects of anti-CCR4 on the RENCA TAMs. BALB/c mice were injected with RENCA-luc cells and treated with Affi-5 ( $T$ ) or isotype control (C). Mice were sacrificed 17 days after surgery, and tumors were dissociated and characterized by flow cytometry. (A) Tumor-infiltrating macrophages (gated as CD45+CD11b+F4/80+) per milligram of tumor for 5 independent experiments are shown. There was no significant difference between Affi-5-treated $(T)$ and isotypetreated (C) tumors ( $n=15$ and $n=14$, respectively). (B and C) Geometric mean of fluorescence intensity (MFI) for MHCII and MR staining on macrophages for 5 independent experiments, and staining for isotype-treated and Affi-5-treated dissociated tumors for 1 representative experiment. Two-tailed Student's $t$ test, ${ }^{* *} P=0.0008$ and ${ }^{* *} P=0.0085$. $n=19$ for $C, n=17$ for $T$. (D) RNA was extracted from macrophages (CD45+CD11 $b^{+} F 4 / 80^{+}$) sorted by flow cytometry from dissociated tumors. The ratio between arginase and Nos2 expression was determined by real-time PCR in 2 independent experiments pooled together (Mann-Whitney $U$ test, ${ }^{*} P=0.035$ ), with $n=6$ for $C, n=7$ for $T$. (E) Cells were dissociated at end point from dissected tumors from BALB/c mice treated with Affi- 5 (T) or isotype control (C) and plated overnight in the presence of brefeldin $A$. The fold change in the number of macrophages (CD45+CD11b $\left.\mathrm{b}^{+} 4 / 80^{+}\right)$positive for intracellular TNF- $\alpha$ is shown from 2 pooled experiments (Mann-Whitney $U$ test, ${ }^{* *} P=0.002, n=11$ for $C$ and $n=15$ for $T$ ). ure 3F). Affi-5 did not influence the growth or viability of either human or murine RCC cells in normal or low serum or their release of CCR4 ligands (data not shown).

RENCA cells labeled with luciferase were grown orthotopically in their syngeneic hosts, WT BALB/c mice, by injection into the renal capsule of the left kidney. Mice reached humane end point during the course of tumor growth between 17 and 21 days after implantation. Anti-CCR4 antibody treatment significantly inhibited tumor burden as measured by tumor weight and bioluminescence as compared with treatment with an isotype control antibody (Figure 2 , B and C). Figure $2 \mathrm{~B}$ shows the mean tumor weights at end point from 6 independent experiments with 20 $\mathrm{mg} / \mathrm{kg}$ Affi-5 versus an isotype control, while Figure 2C shows a typical experiment using bioluminescence as a measure of tumor growth. 10 $\mathrm{mg} / \mathrm{kg}$ Affi-5 also had significant antitumor activity (Figure 2D). There was a significant reduction in the serum concentration of the CCR4 ligand CCL17 in treated mice obtained for A498 (data not shown) and murine RENCA cells (Supplemental Figure 3, A and B), with characteristic bell-shaped concentration-response curves typical of chemokine-mediated migration, implying that the receptor can be functional on malignant cells. shRNA to CCR4 abolished CCR4 staining in the RCC cells as well as their migration to CCL17 (Supplemental Figure 3, $\mathrm{C}$ and D). In addition, a fully human anti-CCR4 antagonistic antibody, Affi-5 (19), abrogated the migration of 786-O cells to CCL17, further confirming that the CCR4 receptor is functional in the tumor cells (Supplemental Figure 3E).

Antitumor activity of an anti-CCR4 antibody. Taken together, the above data and the published literature suggest that CCR4 is a therapeutic target of interest in human solid cancers, especially as it is expressed by both malignant cells and tumor-infiltrating leukocytes. We therefore conducted preclinical experiments using the Affi-5 antagonistic antibody to CCR4 in the RENCA mouse RCC model. Affi- 5 inhibited migration of RENCA cells to murine CCL17 and CCL22, showing that the antibody was antagonistic to murine CCR4 as well as human CCR4 (Figure 2A and Supplemental Fig-
(Figure 2E). In contrast, the serum concentration of CCL22 was low and did not change following treatment (Figure 2F). Analysis of CCL17 and CCL22 expression in tumor lysates showed that, adjusted for tumor size, CCL17 levels were stable while CCL22 levels were higher in anti-CCR4-treated tumors (data not shown), suggesting that the decrease in CCL17 circulating levels might reflect a reduction in tumor size.

Actions of the anti-CCR4 antibody on TAMs. We considered based on the data presented previously, as well as the published literature, that the mechanism of anti-CCR4 inhibition on tumor growth could involve direct effects on malignant cells and/or on leukocytes. To investigate mechanisms of action of the antiCCR4 antibody, we studied single-cell suspensions from the treated tumors. Several cell types were positive for CCR4 staining in control tumors: macrophages $\left(\mathrm{CD} 45^{+} \mathrm{F} 4 / 80^{+} \mathrm{CD} 11 \mathrm{~b}^{+}\right)$and different $\mathrm{T}$ cell subtypes, such as $\mathrm{CD} 4^{+}\left(\mathrm{CD} 45^{+} \mathrm{CD} 3^{+} \mathrm{CD} 4^{+} \mathrm{FoxP}^{-}\right)$, $\mathrm{CD} 8^{+}$lymphocytes $\left(\mathrm{CD} 45^{+} \mathrm{CD}^{+} \mathrm{CD}^{+}\right)$, and Tregs $\left(\mathrm{CD} 45^{+} \mathrm{CD}^{+}\right.$ $\mathrm{CD}^{+}{ }^{+} \mathrm{FoxP}^{+}$) (Supplemental Figure 4 ). NK cells were also weakly positive for CCR4 staining (Supplemental Figure 4). Compared 
A

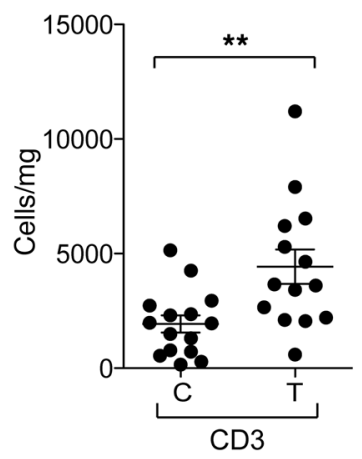

D

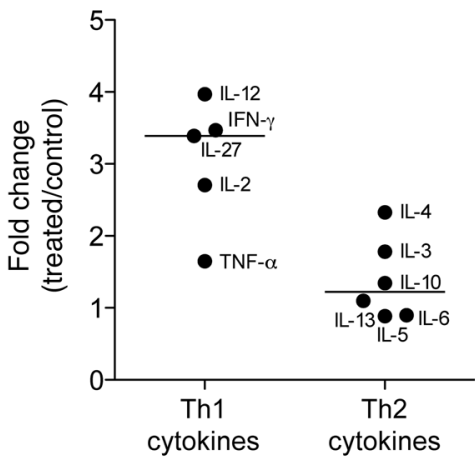

B

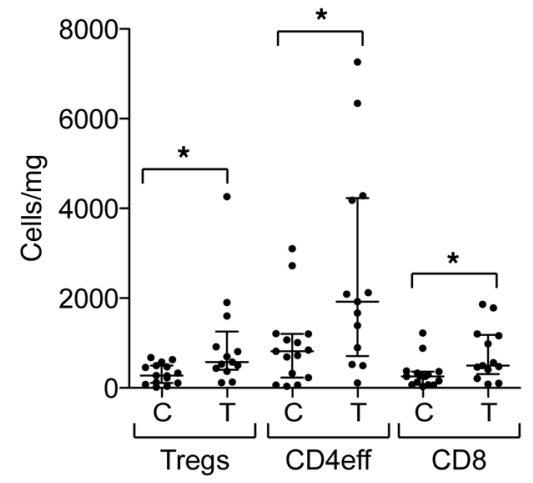

C

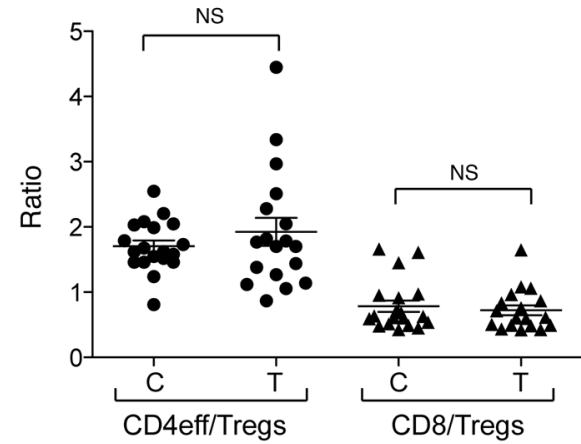

E

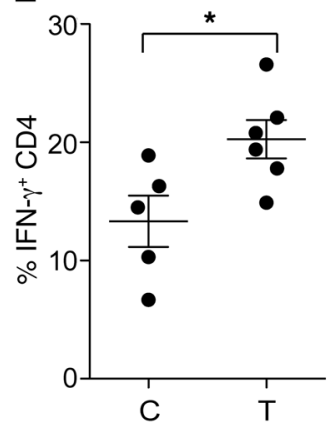

$\mathbf{F}$

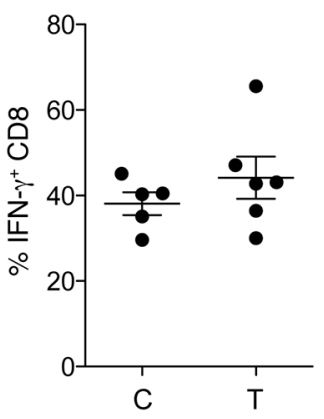

G

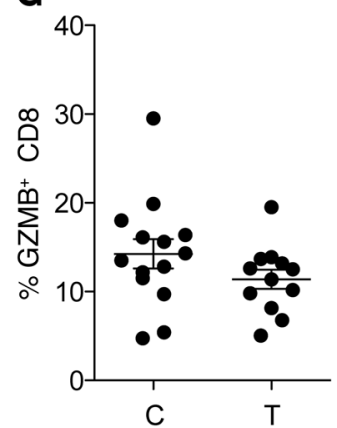

Figure 4. Involvement of T cells in the actions of the anti-CCR4 antibody. BALB/c mice were injected with RENCA-Iuc cells and treated with Affi-5 (T) or isotype control (C). Mice were sacrificed 17 days after surgery, and tumors were dissociated and characterized by flow cytometry. (A and B) Number of $\mathrm{CD} 5^{+} \mathrm{CD3}^{+}$(A) and CD4+FoxP3+ (Tregs), CD4 ${ }^{+}$FoxP3- (CD4eff), and CD8+ (B) cells/mg of tumor in tumors from isotype-treated (C) or Affi-5-treated (T) mice for 4 experiments pooled together. Two-tailed Student's $t$ test, $(\mathbf{A}){ }^{*} P=0.003$ and $(\mathbf{B}){ }^{*} P=0.01$ (Tregs and CD8), ${ }^{*} P=0.02$ (CD4eff), with $n=15$ for C, and $n=14$ for $\mathrm{T}$ ). (C) Ratio of $\mathrm{CD}^{+}{ }^{+} \mathrm{CD} 4^{+}$FoxP3 $3^{-}$or $\mathrm{CD}^{+}{ }^{+} \mathrm{CD} 8^{+}$to $\mathrm{CD3}^{+} \mathrm{CD} 4^{+}$FoxP3 $3^{+}$(Treg) lymphocytes in isotype-treated and Affi-5-treated mice for 4 experiments. (D) Two control-treated (control) and 2 Affi-5-treated (treated) tumors from BALB/c mice were lysed, and an amount equivalent to $200 \mu$ g tumor lysate was incubated on Proteome Profiler Mouse Cytokine Array Panel A membranes. Average signal for each cytokine was normalized to signal from control-treated tumors. Fold change compared with control, grouped for Th1 cytokines and Th2 cytokines, is shown (2-tailed Student's $t$ test, $P=0.0173$ ). (E and F) CD3 cells were isolated at end point from dissected tumors from BALB/c mice treated with Affi-5 (T) or isotype control (C). Lymphocytes were stimulated with PMA and ionomycin for 4 hours in the presence of brefeldin A and stained for intracellular IFN- $\gamma$. The percentage of IFN- $\gamma-$ positive CD4 ${ }^{+}$cells (E) or CD8 ${ }^{+}$cells (F) is represented. Two independent experiments pooled together are shown (2-tailed Student's $t$ test, ${ }^{*} P=0.028$ for IFN- $\gamma$-positive CD4 ${ }^{+}$cells [E], $n=5$ for $C, n=6$ for T). (C) Percentage of $C D 8^{+}$cells positive for granzyme $B(G Z M B)$ is represented, from 4 independent experiments.

with the leukocyte populations, $\mathrm{CD}^{-} 5^{-}$cells, which contain the malignant cell population, were only weakly positive for CCR4 (Supplemental Figure 4).

As TAMs showed expression of CCR4, we first studied the numbers and phenotype of these cells. The number of TAMs (gated as $\mathrm{CD} 45^{+} \mathrm{CD} 11^{+} \mathrm{CD} 11 \mathrm{~b}^{+} \mathrm{F} 4 / 80^{+}$) per milligram of tumor did not differ significantly between Affi-5-treated and control-treated tumors (Figure 3A). However, the phenotype of the macrophages was affected by treatment. TAMs from Affi-5-treated mice expressed significantly higher levels of MHC class II (MHCII) and lower levels of mannose receptor (MR) compared with TAMs from controltreated mice (Figure 3, B and C). In the spectrum of phenotype of macrophage activation, low MHCII expression and high MR levels are associated with an $\mathrm{M} 2$ phenotype, which promotes tissue repair and cell proliferation (20). Conversely, high MHCII expression and low MR expression are characteristic of an M1 macrophage phenotype, associated with antitumor activity. To further characterize the TAM phenotype, we extracted mRNA from macrophages from Affi5- and control-treated tumors and analyzed them for arginase 1 and inducible Nos 2 expression. The arginase/Nos 2 expression ratio was significantly lowered by anti-CCR 4 treatment (Figure 3D). Purified Affi-5-treated macrophages also showed increased intracellular TNF, further evidence for an M1 cytotoxic phenotype (Figure 3E). Taken together, these data imply that the phenotype of macrophages from anti-CCR4-treated tumors was altered compared with isotype control-treated tumors and displayed several characteristics associated with an antitumor response.

We next asked whether CCR4 inhibition could have similar activities in another mouse cancer model, testing Affi-5 in the B16 melanoma model. As shown in Supplemental Figure 5, treatment altered the TAM phenotype, with a significant increase in MHCII in myeloid cells in 2 separate experiments (Supplemental Figure $5 \mathrm{~A}$ ). There was, however, no effect on MR expression (Supplemental Figure 5B) or tumor weight (Supplemental Figure 5C) in this rapidly growing model.

Actions of the anti-CCR4 antibody on $T$ cells in the tumor microenvironment. We next examined changes among the $\mathrm{CD}^{+}$ $\mathrm{T}$ cells, which were also positive for CCR4 in the RENCA tumor 
A

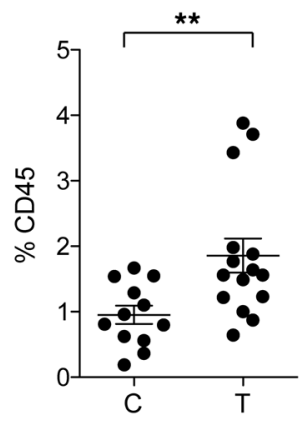

B

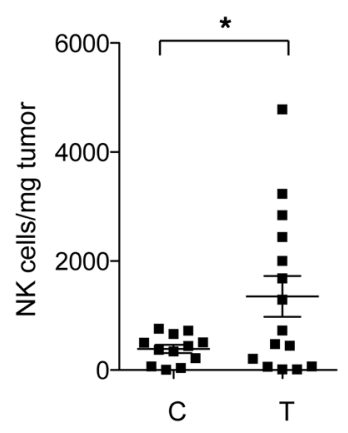

C

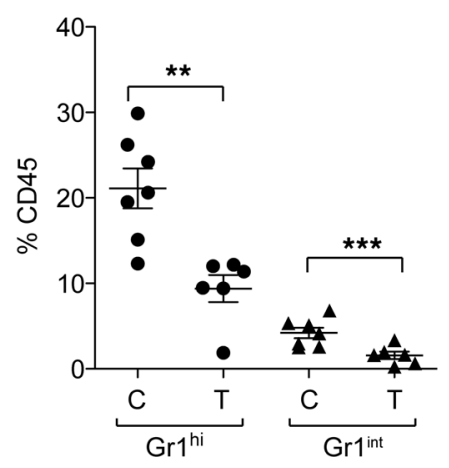

D

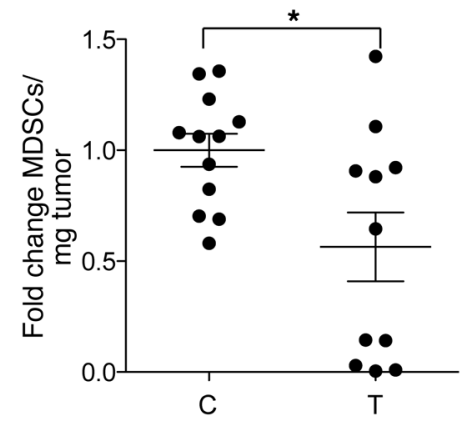

E

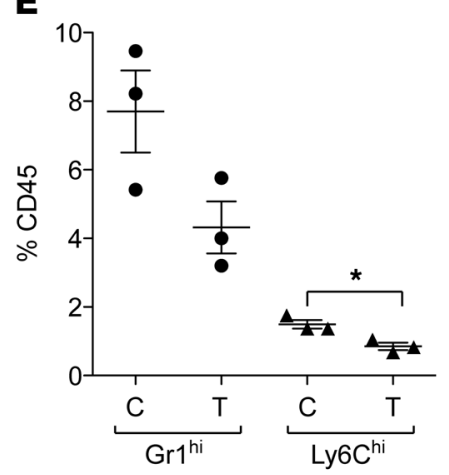

$\mathbf{F}$

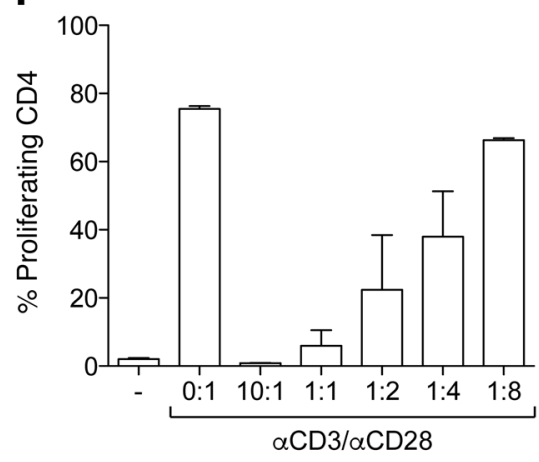

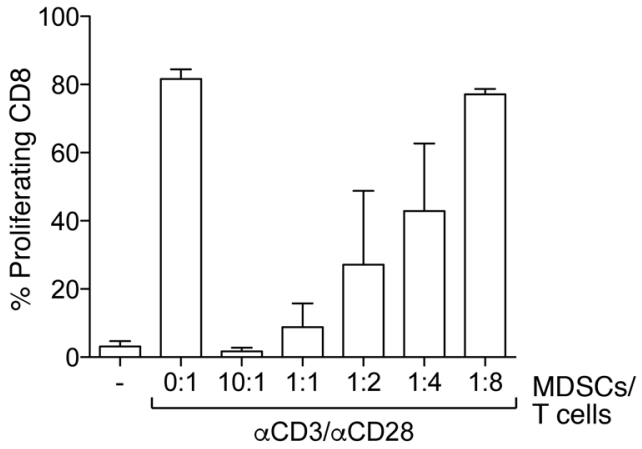

Figure 5. Other effects of anti-CCR4 antibody in the RENCA tumor model. BALB/c mice were injected with RENCA-luc cells and treated with Affi-5 (T) or isotype control (C). Mice were sacrificed 17 days after surgery, and tumors or spleens were dissociated and characterized by flow cytometry. (A) Percentage of NK cells $\left(C D 45^{+} C D 3^{-D X 5^{+}}\right.$) among the $C D 45^{+}$population in tumors; 2 -tailed Student's $t$ test, ${ }^{* *} P=0.0064,4$ independent experiments pooled together ( $n=12$ for C, $n=15$ for T). (B) The number of NK cells/mg of tumor was also significantly higher with treatment (2-tailed Student's $t$ test, ${ }^{*} P=0.032$, $n=12$ for $C, n=15$ for $T$ ). (C) Percentage of MDSCs (CD45+CD11+C $r 1^{+}$) among the CD45+ infiltrate for tumors from control and treated animals. Two populations of MDSCs ( $G r^{\text {hi }}$ and $\left.G r^{\text {int }}\right)$ were identified and analyzed separately. Two-tailed Student's $t$ test, ${ }^{* *} P=0.0021$ and ${ }^{* * *} P=0.0065 ; 2$ experiments pooled together, $n=7$ for $C$ and $n=6$ for T. (D) The fold change in the number of MDSCs/mg of tumor was also significantly lower in the tumors from treated animals (4 experiments, 2-tailed Student's $t$ test, ${ }^{*} P=0.017, n=12$ for $C, n=11$ for T). (E) Percentage of MDSCs (CD45+CD11b+C $1^{+}$) among the CD45+ infiltrate

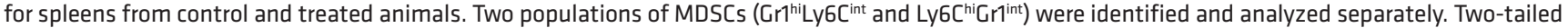
Student's $t$ test, $P=0.075$ for $\mathrm{Gr}^{\mathrm{hi}},{ }^{*} P=0.018$ for Ly6C hi,$n=3$. (F) Naive CD3 ${ }^{+}$cells isolated from spleen of healthy mice $\left(5 \times 10^{4} /\right.$ well $)$ were pre-labeled with CFSE and activated with anti-CD3- and anti-CD28-coated beads at a ratio 1:2 beads/CD3 cells in the presence of freshly isolated MDSCs from tumors. Cells were cocultured for 3 days, and CD4 ${ }^{+}$and CD8 ${ }^{+} T$ cell proliferation was measured by CFSE dye dilution from 2 independent experiments, each pooling MDSCs from 2-3 tumors. Proliferation was inhibited significantly (1-way ANOVA, $P=0.0012$ for CD4, $P=0.0058$ for CD8, at MDSC/T cell ratios of 10:1, 1:1, 1:2).

microenvironment. The number of $\mathrm{CD}^{+}$cells per milligram of tumor was significantly higher in Affi-5-treated tumors compared with controls (Figure 4A). As CCR4 has been implicated in the recruitment of Tregs at tumor sites $(12,13)$, we hypothesized that treatment with anti-CCR4 would reduce the number of CCR4 $4^{+}$Tregs, as observed in adult $\mathrm{T}$ cell leukemia patients treated with mogamulizumab (18). However, the number of Tregs per milligram of tumor was higher in anti-CCR4-treated tumors (Figure $4 \mathrm{~B}$ ). Also the number of $\mathrm{CD}^{+}$effector cells and $\mathrm{CD}^{+}$cells per milligram of tumor was increased with treatment (Figure 4B). As a result of these changes, the ratios of $\mathrm{CD} 4^{+} \mathrm{T}$ effector or $\mathrm{CD}^{+} \mathrm{T}$ cells to Tregs in tumors were unaffected by Affi-5 treatment (Figure 4C).

However, there was an increase in the amount of Th1 cytokines compared with Th2 cytokines in the Affi-5-treated tumor lysates (Figure 4D), which could help explain the M2/M1 switch observed in the TAMs.

We next explored in more detail the phenotype of $\mathrm{CD}^{+}$ effector and $\mathrm{CD}^{+}$cells. The number of $\mathrm{CD}^{+}$cells positive for IFN- $\gamma$ expression was increased in treated tumors (Figure $4 \mathrm{E}$ ), while it was unaltered for the $\mathrm{CD}^{+}$cells (Figure $4 \mathrm{~F}$ ). Moreover, CD8 staining for granzyme $\mathrm{B}$ was not significantly altered by treatment (Figure 4G). Collectively, these data suggested a role for $\mathrm{CD} 4^{+}$cells in the antitumor activity of the anti-CCR 4 antibody.

Other treatment-induced changes to tumor-infiltrating leukocyte populations. We observed repeatedly a significant increase in tumor NK cells as a proportion of total $\mathrm{CD} 45^{+}$cells (Figure $5 \mathrm{~A}$ ), while the proportion of granzyme B-positive NK cells was not altered (data not shown). The effects on NK cells was due to changes in cell number, as a significant increase in terms of NK cells/mg tumor could be observed $(P=0.03)$ (Figure $5 \mathrm{~B}$ ). Also, myeloid-derived suppressor cells (MDSCs), characterized as $\mathrm{CD} 45^{+} \mathrm{CD} 11 \mathrm{~b}^{+} \mathrm{Gr} 1^{+}$, constituted a reduced percentage of the $\mathrm{CD} 45^{+}$infiltrate in tumors from treated compared with control mice (Figure $5 \mathrm{C})$, as there were fewer granulocytic $\left(\mathrm{Gr}^{\mathrm{hi}}\right)$ and monocytic (Gr1 $\left.{ }^{\text {int }}\right)$ MDSCs in treated tumors. As for the MDSCs, there was some variability between experiments in terms of numbers of cells, so we expressed the results as fold change in cells/ 
A

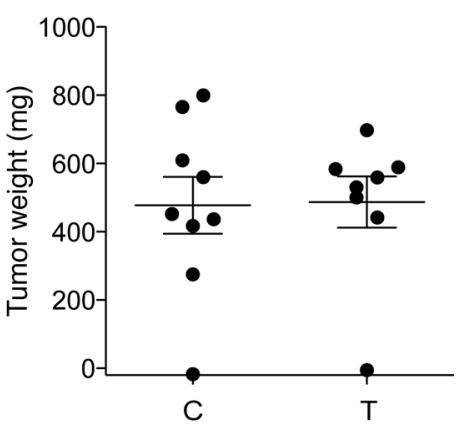

E

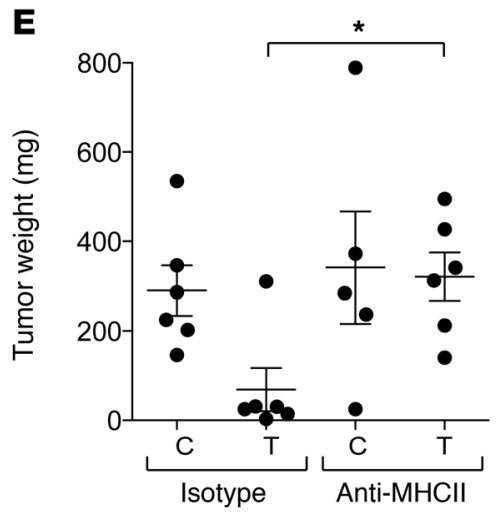

B

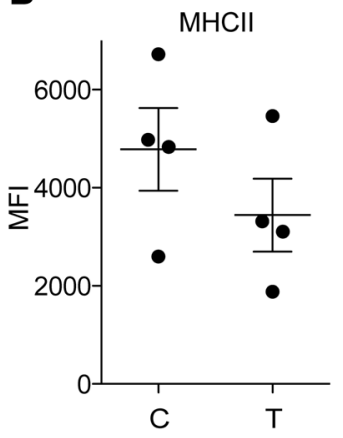

$\mathbf{F}$

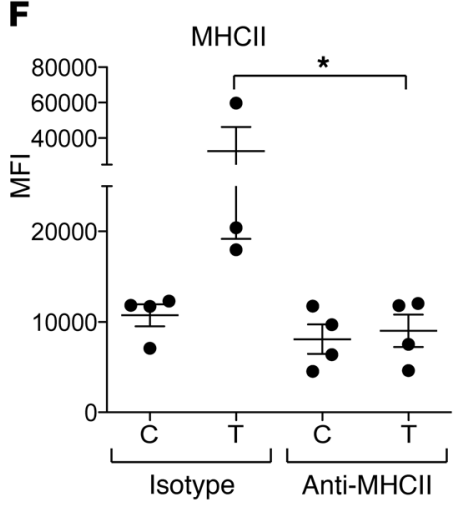

C

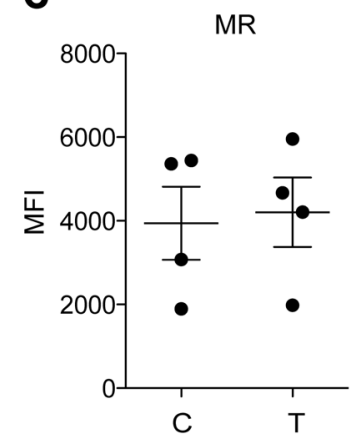

D

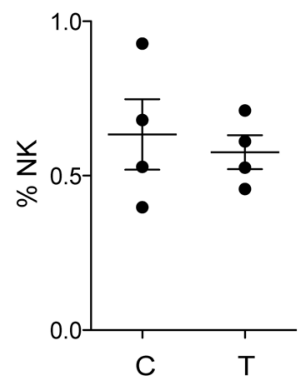

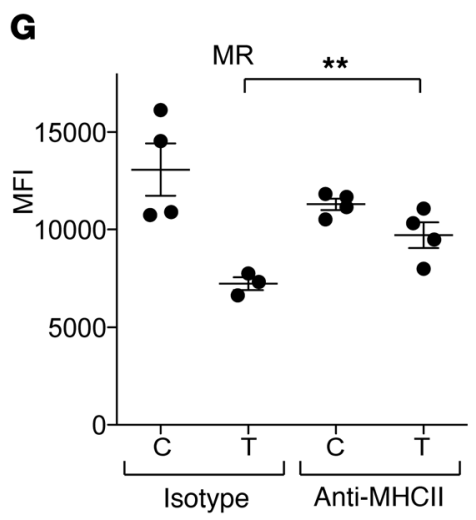

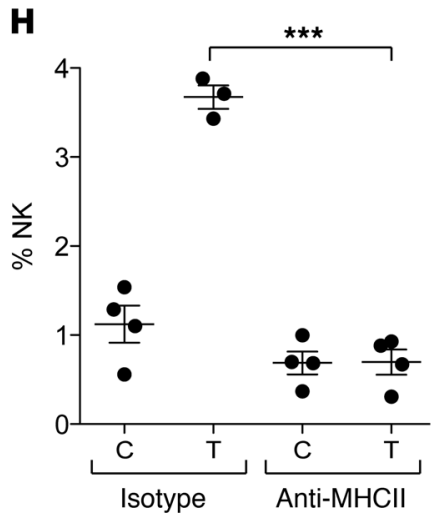

Figure 6. Effects of anti-CCR4 on the RENCA tumors require CD4+ cells. (A-D) BALB/c nu/nu mice were injected with $1 \times 10^{5}$ RENCA-luc cells and treated with Affi-5 (T) or isotype control (C) (10 mg/kg) twice weekly starting 48 hours after surgery. Mice were sacrificed at 17 days after surgery, and tumor weight was determined ( $n=9 \mathrm{C}, n=8 \mathrm{~T}$, not significant). Ceometric mean of fluorescence intensity for MHCII (B) and MR (C) staining on macrophages $\left(C D 45^{+} C D 11 b^{+} F 4 / 80^{+}\right)$, for isotype-treated and Affi-5-treated dissociated tumors; $n=4$. (D) Percentage of NK cells $\left(C D 45^{+} C D 3^{-} D X 5^{+}\right)$among the CD45 population; $n=4$. (E-H) BALB/c mice were injected with $1 \times 10^{5}$ RENCA-luc cells and treated with Affi-5 (T) or isotype control (C) (10 mg/kg) twice weekly starting 48 hours after surgery. Treatment with anti-MHCII or the relevant isotype control $(10 \mathrm{mg} / \mathrm{kg})$ was started 1 day prior to surgery and continued with 3 doses per week. Mice were sacrificed 17 days after surgery, tumor weight was determined ( $n=6$ for each group), and tumors were dissociated and characterized by flow cytometry. (E) Blocking of $\mathrm{MHCII}$ has a significant effect on tumor weight (2-way ANOVA, $\left.{ }^{*} P=0.049\right)$. Bonferroni post-test showed significant difference $(P<0.05)$ in weight of Affi-5-treated tumors in the presence versus absence of anti-MHCII. (F and $\mathbf{G})$ Geometric mean of fluorescence intensity (MFI) for MHCII (F) and MR (G) staining on macrophages (CD45+CD11b+F4/80+). There is a significant difference between MHCII and MR expression of macrophages from Affi-5-treated tumors in the presence or absence of anti-MHCII (Kruskal-Wallis test with Dunn post-test, ${ }^{*} P<0.05$ and 1-way ANOVA with Bonferroni post-test, ${ }^{* *} P<0.001$, with $n=3-4$ for each group). (H) Percentage of NK cells (CD45 ${ }^{+}$CD3-DX5 ${ }^{+}$) among the CD45 ${ }^{+}$population. There is a significant difference (1-way ANOVA with Bonferroni post-test, $\left.{ }^{* *} P<0.001\right)$ in the percentage of NK cells from Affi-5-treated tumors in the presence versus absence of anti-MHCII.

mg tumor. Pooling results from 4 experiments, we see a significant reduction in treated tumors $(P=0.017)$ (Figure 5D). A similar reduction of granulocytic (Gr1 ${ }^{\text {hi }}$ ) and monocytic (Ly6-C $\left.\mathrm{C}^{\text {hi }}\right)$ MDSCs was seen in the spleen of treated mice compared with control mice (Figure 5E). To determine whether the accumulated CD11b+Gr1 ${ }^{+}$ cells have a suppressive phenotype and could really be identified as MDSCs, we performed immunosuppression assays using $\mathrm{T}$ cells as effectors. Increasing amounts of MDSCs from the tumors of untreated mice effectively suppressed proliferation of activated $\mathrm{CD}^{+}$and $\mathrm{CD} 8^{+}$cells isolated from naive splenocytes (Figure $5 F)$. Similarly, splenic MDSCs isolated from tumor-bearing mice suppressed proliferation of $\mathrm{CD} 4^{+}$and $\mathrm{CD} 8^{+}$cells (data not shown). 

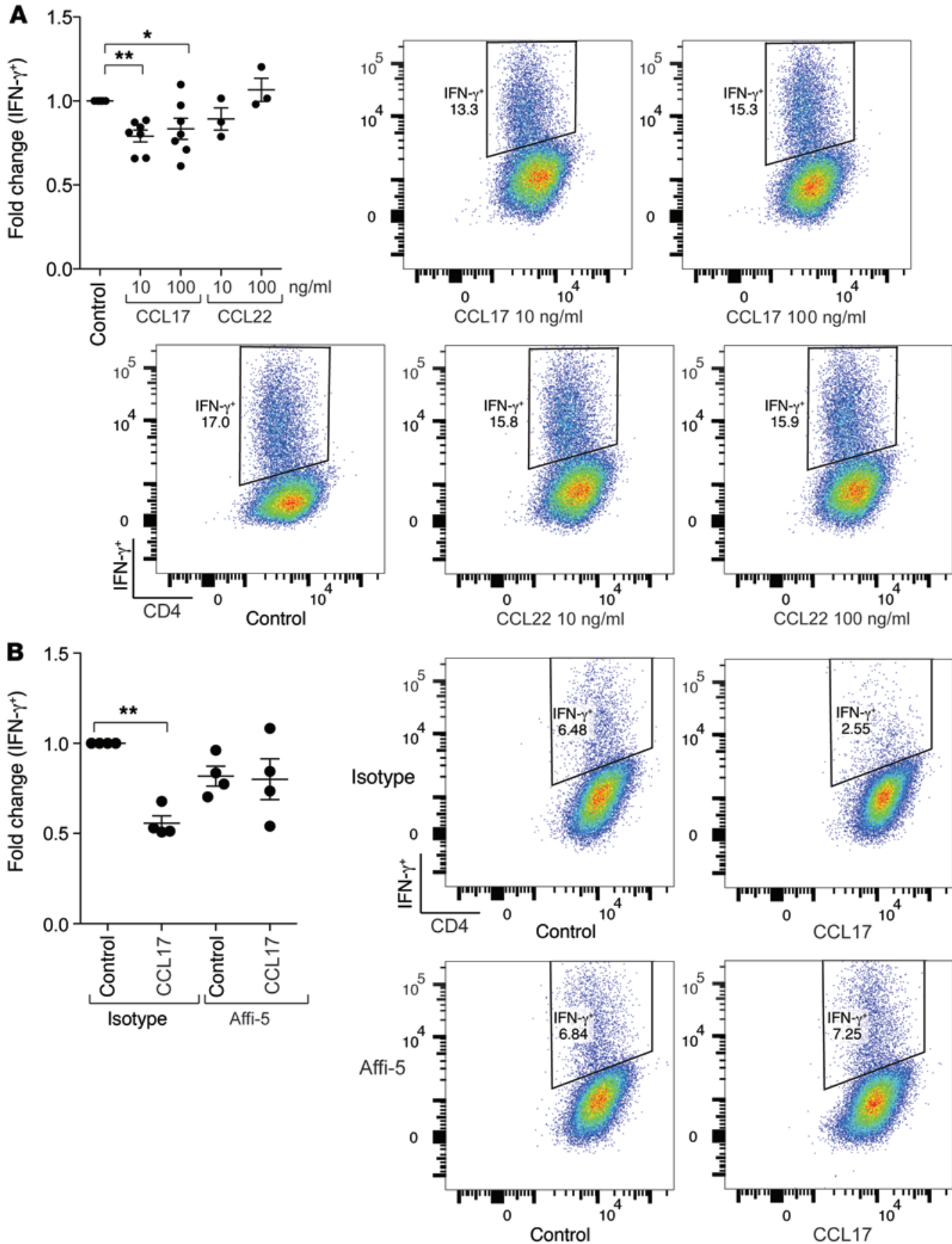

Figure 7. CCL17 can inhibit Th1 responses in vitro. (A and $\mathbf{B}) \mathrm{CD}^{+}$ cells were isolated from spleens of healthy mice and stimulated with IL-2 and IL-12 in the presence of anti-CD3- and anti-CD28-coated beads. CCL17, CCL22, Affi-5 (10 $\mu \mathrm{g} /$ $\mathrm{ml})$, or isotype control was added after an overnight incubation; after 3 days cells were stimulated with cell stimulation cocktail, harvested, stained for intracellular IFN- $\gamma$, and analyzed by flow cytometry. Results of 7 and 3 independent experiments are shown for CCL17 and CCL22, respectively (A), together with representative plots of 1 experiment. Results of 4 independent experiments are shown in B, with representative plots of 1 experiment. ${ }^{*} P<0.05$ ${ }^{*} P<0.01$, 1-way ANOVA with Bonferroni post-test.
Further investigation of CCR4 receptor on tumor $C D 4^{+} T$ cells. As in our model we observed changes to cells of both the adaptive and innate immune response, and some of our evidence pointed to involvement of $\mathrm{CD} 4^{+} \mathrm{T}$ cells, we wanted to better understand the interplay between these different components. Affi- 5 did not inhibit RENCA tumor growth in T cell-deficient nude mice (Figure 6A), and there was also no effect on macrophage phenotype or extent of NK cell infiltrate in tumors in nude mice (Figure 6, B-D). This suggested that adaptive immunity, especially via $\mathrm{CD} 4^{+} \mathrm{T}$ cells, was upstream of the actions on the cells of the innate immune system.

To further investigate a role for $\mathrm{CD}^{+} \mathrm{T}$ cells in the antitumor actions of Affi-5, we combined this agent with a neutralizing antibody to MHCII. This completely abrogated the effects of the anti-
CCR4 antibody on RENCA tumor weight (Figure 6E), macrophage MHCII (Figure 6F), and MR expression (Figure 6G), and percentage of NK cells in the tumor microenvironment (Figure 6H).

We concluded that $\mathrm{CD} 4^{+} \mathrm{T}$ cells are essential mediators of the actions of the anti-CCR4 receptor antibody Affi-5, and were required for the observed changes in macrophage phenotype and proportion of NK cells.

CCR4 function in normal $C D 4^{+} T$ cells. Our results led us to question whether CCR4 might be involved in direct regulation of $\mathrm{Th} 1$ and $\mathrm{Th} 2$ responses in normal $\mathrm{CD} 4^{+} \mathrm{T}$ cells. To explore this, we developed an in vitro assay in which $\mathrm{CD} 4^{+} \mathrm{T}$ cells were purified from splenocytes of healthy mice and polarized to a Th1 response with IL-12 and IL-2 in the presence of anti-CD3 and anti-CD28 
A

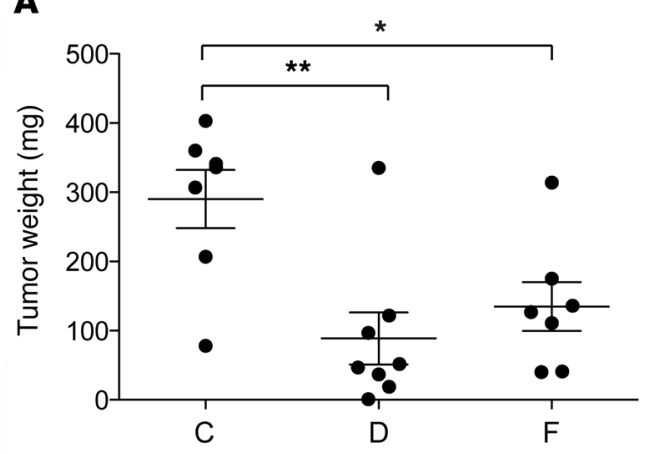

B

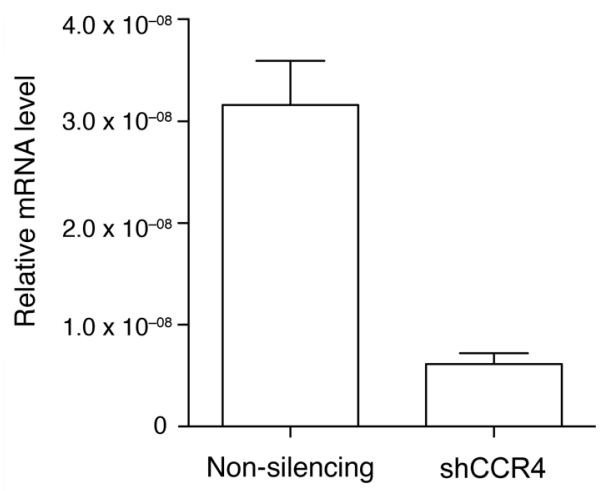

C

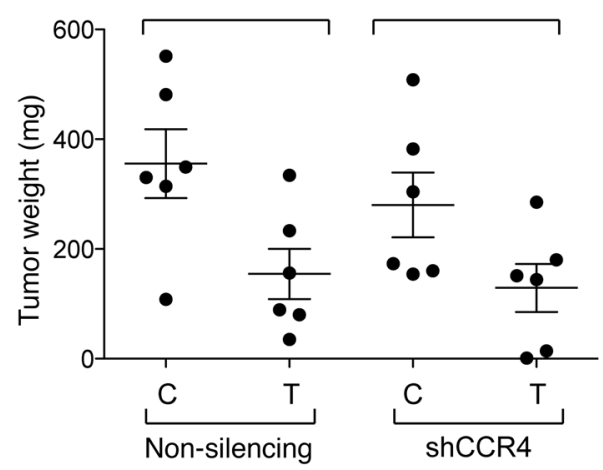

Figure 8. Effects of anti-CCR4 antibody are not dependent on malignant cell expression of CCR4. (A) BALB/C mice were injected with $1 \times 10^{5}$ RENCA-luc cells and treated with defucosylated Affi-5 (T; D), fucosylated Affi-5 (T; F), or defucosylated isotype control (C) at $10 \mathrm{mg} / \mathrm{kg}$ twice weekly starting 48 hours after surgery. Mice were sacrificed 17 days after surgery, and tumor weight was analyzed ( $n=7 \mathrm{C}, n=8 \mathrm{~T}$ [D], $n=7 \mathrm{~T}$ [F]). Two-tailed Student's $t$ test, ${ }^{* *} P<0.01,{ }^{*} P<0.05$. (B) RENCA-luc cells were infected with lentivirus encoding for non-silencing shRNA, or anti-CCR4 shRNA. Silencing of the infected cells lines was verified at the RNA level by real-time PCR. (C) BALB/c mice were injected with $1 \times 10^{5}$ RENCA-luc cells infected with a non-silencing lentivirus, or CCR4 shRNA (shCCR4), and treated twice weekly with Affi-5 at $10 \mathrm{mg} / \mathrm{kg}$ (T) or isotype control (C), starting 48 hours after surgery; $n=6$ for each group. Mice were sacrificed 17 days after surgery, and tumor weight was recorded. Affi-5 treatment had an effect (2-way ANOVA, $P=0.0036)$, and CCR4 silencing did not interact significantly with the Affi-5 treatment $(P=0.64)$.

beads. This treatment stimulated production of IFN- $\gamma$ over the course of 3 days. When CCL17 was added to the CD $4^{+}$cells 1 day after the initial stimulation, a significant reduction in the production of IFN- $\gamma$ was observed (Figure 7A). Although CCL22 produced a similar trend, it was markedly weaker than CCL17 in inhibiting Th1 polarization (Figure 7A). The action of CCL17 on CD4 $4^{+}$cells in vitro was abolished by addition of the anti-CCR4 antibody (Figure 7B). These results indicate that CCL17 might play a role in directly inhibiting the Th1 response, and provide more mechanistic insight into the action of Affi-5. CCL17 in the tumor microenvironment might be secreted by many cell types, including M2polarized macrophages (21). To support this hypothesis, we measured mRNA levels for Ccl17 and Ccl22 in the different cell populations of the tumor microenvironment. Macrophages showed the highest expression of the two chemokines (Supplemental Figure 6), although a contribution from other cell types cannot be excluded.

Is ADCC involved in the antitumor action of the anti-CCR 4 antibody? Finally, as ADCC is implicated in the mechanisms of action of the anti-human CCR4 antibody currently used clinically in treatment of hematological malignancies (16), we investigated the role of ADCC in the actions of Affi-5. While it is possible that murine $\mathrm{Fc}$ receptors would interact with a human antibody (22), both a defucosylated (which was used in all the experiments presented up to this point) and a fucosylated version of Affi- 5 antibody had similar and significant antitumor effects (Figure 8A). Moreover, the CCR4 antagonist Affi-5 retained antitumor activity on RENCA cells in which CCR4 was silenced by shRNA (Figure 8, B and C). This result indicates that the antitumor effect of Affi- 5 occurs primarily through modulation of non-malignant cells in the tumor microenvironment. This is not entirely unexpected, since in vivo $\mathrm{CD}^{-} 5^{-}$cells, which include RENCA cells, expressed low levels of CCR4 (Supplemental Figure 4). However, as this antibody has a reported ADCC activity against human lymphoma cells (19), it may act by ADCC on other tumor cells where CCR4 expression is higher.

Levels of PD-L1 and CTLA4 after treatment. Immune checkpoint targeting has proven to be a promising approach in the treatment of RCC. Therefore, we investigated whether the anti-CCR4 treatment had an impact on immune checkpoint ligands. As shown in Supplemental Figure 7, CTLA4 expression on Tregs, and PD-L1 expression on macrophages, $\mathrm{CD}^{+} \mathrm{T}$ cells, and $\mathrm{CD} 45^{-}$cells, was retained after anti-CCR4 treatment. This would suggest that, as the anti-CCR4 treatment displays actions on different components of the immune infiltrate, it could be a good candidate to be administered together with promising immune checkpoint inhibitors.

\section{Discussion}

In this study, we provide a comprehensive analysis of the expression of the chemokine receptor CCR 4 and its ligands CCL17 and CCL22 in a solid tumor. We present evidence that CCR4 is expressed at significant levels in renal cancer biopsies, where it is associated with the extent of immune infiltrate. Also, expression of CCL17 and CCL22 is altered in renal cancer tissue and in the plasma of patients. In fact, a high CCL17/CCL22 ratio in plasma is associated with a worse prognosis. This is reminiscent of what has been observed in other solid tumors, where there is high CCR4 expression that is generally associated with a poor prognosis $(14,15)$. CCL22 is also detected in the tumor microenvironment of ovarian, hepatocellular, and breast cancer $(10,12,13)$. Our work is further supported by a recent multivariate analysis of CCR4 expression in 53 RCC patient 
biopsies, where CCR4 expression was an independent risk factor for poor prognosis and overall survival (23). Taken together, these observations suggest that CCR 4 is an attractive therapeutic target in solid cancers.

In the current work, we have not considered a role for the chemokines CCL2 and CCL5, which may also bind to CCR4, as CCL17 and CCL22 have the highest affinity for the receptor, but in future studies it would be interesting to assess the effect of CCR4 inhibitions on their local and systemic levels.

In this study we report for the first time to our knowledge that an anti-CCR4 antibody has activity in a solid cancer model. As CCR 4 is expressed on a number of different immune cells, we had expected that the CCR4 antibody would reduce the number of tumor-infiltrating leukocytes as part of its mechanism of action, but this did not occur. In particular, we did not observe an effect on the number of infiltrating Tregs, which are thought to be recruited though CCR4 in the tumor microenvironment of different tumor types (11-13). In fact, the antibody caused unexpected changes in the phenotype of myeloid cells in the RENCA tumor microenvironment from potentially pro- to antitumor. TAMs mainly consist of a population with little cytotoxicity for tumor cells because of their limited production of $\mathrm{NO}$ and proinflammatory cytokines. At the same time, TAMs also possess poor antigen-presenting capability (20). This has led to the notion that depleting TAMs from the tumor microenvironment is an interesting target in cancer therapy. However, it was shown recently that inhibition of the macrophage cell surface receptor CSF1R with a small molecule inhibitor in a model of glioblastoma was able to reduce tumor progression by reducing the M2 polarization of TAMs (24). This work proved that modification of TAM tumor-promoting functions may have a significant impact on tumor growth and that depletion is not strictly necessary for an effective TAM-targeted therapy. In our work, the use of an anti-CCR4 antibody achieved, through a different mechanism of action, a similar change of TAM phenotype, which resulted in a reduction of tumor growth. Findings in human renal cancer provide an interesting correlate to our experimental data, suggesting that stimulating an M2/Th2 to M1/Th1 response may be of therapeutic value. In a recent analysis of the intratumoral immunologic profile of RCC biopsies, the expression of M2 macrophage markers correlated with a poor prognosis and high tumor NOS2 mRNA levels with a good prognosis (25). To confirm the importance of macrophages in the mechanism of action of Affi-5, a depletion experiment with clodronate liposomes was set up, but the partial depletion of the macrophages was accompanied by an M1 polarization of the remaining macrophages (data not shown), thus invalidating this model for testing our hypothesis. The potential of the anti-CCR 4 antibody was also suggested in the B16 melanoma experiments, where we found an increase in MHCII expression. However, this "partial switch" was not enough to generate an antitumor effect.

As part of the multiple effects of the anti-CCR4 antibody, we also observed a significant increase in NK cells, as well as reduction in MDSCs and circulating CCL17, which can additionally contribute to decreased tumor growth. To determine the contribution of these different populations infiltrating the tumors, we attempted to deplete NK cells and MDSCs in this model. However, while systemic depletion was successful, we never obtained a satisfactory depletion in the tumor microenvironment. As proven by the experiment in nude mice, these multiple effects are not directly mediated by leukocytes of the innate immune system, but are dependent on the adaptive immune system. Moreover, disrupting the MHCII-TCR interaction abolished the therapeutic effect of the anti-CCR4 antibody and also impacted innate immunity, thus proving an essential role for $\mathrm{CD} 4^{+}$cells, linked to their ability to secrete IFN- $\gamma$. Our in vitro assay further supported the hypothesis that the CCR4/CCL17 axis may be involved in maintaining Th2 responses. To our knowledge, the effects described here of CCR4 inhibition in this tumor microenvironment are novel.

We had also predicted that the anti-CCR4 antibody Affi-5 may have ADCC activity in the RENCA model, especially on the malignant cells, but the RENCA cells in vivo had low expression of CCR4. CCR4 is strongly expressed in several human T cell malignancies. Mogamulizumab (KW-0761; Poteligeo), a humanized fucosylated anti-CCR4 antibody that markedly enhances ADCC, is used in the treatment of patients with relapsed or refractory CCR4-positive ATL in Japan (26). Recently it also received approval in Japan for relapsed or refractory CCR4-positive peripheral T cell lymphoma (PTCL) and cutaneous T cell lymphoma (CTCL). We could find no evidence that Affi- 5 was working via ADCC in our model system, but this does not preclude such an action in other models or patients where malignant cells have higher levels of CCR4. Neither, we would suggest, does it preclude mogamulizumab having other actions in the tumor microenvironment. Nor could we find evidence that the anti-CCR4 antibody had direct effects on the CCR4-expressing malignant cells. This may be because the $\mathrm{CD} 45^{-}$population, which contained the malignant cells, expressed low levels of CCR4 in the tumor microenvironment. In addition, RENCA tumors growing in nude mice did not respond to the anti-CCR4 antibody. As anti-CCR4 inhibited RENCA cell migration in vitro, it is possible that the antibody had an antimetastatic effect, but it was not possible to measure this in our model system.

In summary, we have described here a therapeutic strategy to target solid tumors with significant CCR4 expression in the tumor microenvironment. As targeting CCR4 in hematological malignancies has shown manageable side effects, this approach could readily be translated into the clinic. Moreover, this opens the possibility for evaluation of combinations of CCR4 inhibition with other immune-modulatory agents. Inhibition of CCR4 had multiple actions in the RCC experimental tumor microenvironment - but predominantly there was evidence of a Th2/M2 to Th1/M1 switch. CCR4 inhibition also increased MHCII expression on TAMs in the B16 model. As neither Treg infiltration nor CD8 activation was affected and the antibody did not alter levels of CTLA4 and PD-L1 in the renal tumor microenvironment, there is a strong rationale for a combination with immune checkpoint blockade. Also combinations with anti-CD40 agonistic antibodies would be an attractive option, as stimulating macrophage activation in a Th1-skewed environment may increase a host antitumor response.

\section{Methods}

Reagents. Recombinant human chemokines CCL17 (catalog 300-30) and CCL22 (catalog 300-36) were purchased from Peprotech. Recombinant mouse CCL17 (529-TR) and CCL22 (439-MD) were purchased from R\&D Systems. 
RNA isolation and real-time PCR. RNA from sorted macrophages was extracted with the RNeasy Micro Kit (QIAGEN) and amplified with the Ovation PicoSL WTA System V2 (NuGEN). Real-time RT-PCR analysis was performed using TaqMan assays (Applied Biosystems) - CCR4 (Hs99999919_m1), 18S (4310893E), arginase 1 (Mm00475988_m1), Nos2 (Mm00440502_m1) - with the ABI StepOnePlus instrument (Applied Biosystems).

Renal tissue and patient plasma samples. Patients samples were collected from patients who had locally advanced or metastatic RCC, who had progressed after first-line cytokine-based therapy (for locally advanced disease), or who were intolerant to first line cytokine-based therapy (for locally advanced or metastatic disease). The TMA mainly comprised clear cell renal carcinomas (75\%), with some biopsies classified as papillary renal cancer. Clear cell renal carcinomas were identified using standard IHC and carbonic anhydrase IX (CAIX) staining. CCR4 was detected on the malignant cells from 153 of 173 malignant tumors, clear cell and non-clear cell, in our TMA. Controls were agematched individuals with no malignancies.

IHC. Paraffin-embedded sections $(4 \mu \mathrm{m})$ were dewaxed and dehydrated, and antigen retrieval was performed by microwaving sections in Antigen Unmasking Solution (Vector Laboratories, H-3300) for 9 minutes. After blocking with the appropriate serum, samples were incubated overnight at $4^{\circ} \mathrm{C}$ using primary antibodies: CCR4 (ab1669 1:300; Abcam), CCL17 (ab182793 1:100; Abcam), CCL22 (500-P107 1:20; Peprotech), CD3 (A0452, 1:100; Dako), CD68 (M0876 1:50; Dako). Following incubation with a biotinylated secondary antibody (anti-goat, anti-rabbit, or anti-mouse IgG, 1:200; Vector Laboratories) for 30 minutes at room temperature, antigens were revealed with 3,3'-diaminobenzidine (Sigma-Aldrich). Omission of the primary antibody and isotype control antibody were used as negative controls. The scoring for intensity of staining on positive cells was as follows: 0 (no expression), 1 (low expression), and 2 (high expression).

ELISA and Meso Scale Discovery System. Human CCL17 and CCL22 were determined from plasma with Meso Scale Discovery System plates (Human TARC Ultra-Sensitive Kit, K151BGC-1; and Human MDC Ultra-Sensitive Kit, K151BAC; Meso Scale Diagnostic). Mouse CCL17 and CCL22 were determined from plasma or serum with Mouse CCL17/TARC Quantikine ELISA Kit (MCC170) or Mouse CCL22/MDC Quantikine ELISA Kit (MCC220) from R\&D Systems.

Cell culture.786-O and B16FO cells were obtained from ATCC, while RENCA used in the in vivo experiments were a gift from Robert Wiltrout (NCI's Frederick Cancer Research and Development Center, Bethesda, Maryland) or were obtained from ATCC for the in vitro experiments. Cells were incubated at $37^{\circ} \mathrm{C}$ in humidified air with $5 \% \mathrm{CO}_{2} .786-\mathrm{O}$ cells were cultured in RPMI culture media containing $10 \% \mathrm{FBS}, 1 \%$ penicillin/ streptomycin (p/s), and $1 \%$ glutamine. RENCA culture medium was further supplemented with $1 \%$ glutamine and $1 \mathrm{mM}$ sodium pyruvate. Renal cancer cell lines were luciferase labeled for in vivo experiments. B16F0 cells were cultured in DMEM containing 10\% FBS and 1\% p/s.

Migration assays. Chemotaxis was assayed using Falcon PET Cell Culture Inserts, $8-\mu \mathrm{m}$ pore (353182, Becton Dickinson). 786-O cells were seeded in the upper chamber at $1 \times 10^{5}$ in $0.5 \mathrm{ml}$ serum-free RPMI, and $1 \mathrm{ml}$ of medium alone or supplemented with recombinant chemokines was added to the lower chamber. RENCA cells were seeded at $2 \times 10^{5}$ in medium containing $1 \%$ serum. After overnight incubation at $37^{\circ} \mathrm{C}$ and $5 \% \mathrm{CO}_{2}$, cells on the upper surface of the filter were removed and migrated cells on the lower surface were stained with Diff-Quik (Dade Behring). For each insert, the number of migrated cells/field ( $40 \times$ for $786-O$, $20 \times$ for RENCA) was determined. The assays were performed in triplicate.

Anti-CCR4 antibody. Affi-5 is a human IgG1 antibody antagonist of CCR4. This antibody is also referred to as 503 (18). It was produced by transient transfection of expression vectors into HEK293T cells with FuGENE transfection reagent (Promega). The ADCC enhanced (defucosylated) variant was produced using a selective class I $\alpha$-mannosidase inhibitor, kifunensine (Sigma-Aldrich), that was added to the culture medium at a concentration of $100 \mathrm{ng} / \mathrm{ml}$. The antibody was then purified using standard conditions and formulated in $20 \mathrm{mM} \mathrm{PBS}, 145 \mathrm{mM} \mathrm{NaCl}$, $\mathrm{pH}$ 7.2. It was diluted to the required concentration in the same buffer.

In vivo experiments. For the renal model, 6- to 8-week-old female BALB/c or BALB/c $n u / n u$ mice from Charles River Laboratories were injected orthotopically with $1 \times 10^{5}$ luciferaselabeled RENCA cells resuspended in Matrigel (354248, BD Biosciences, Becton Dickinson) into the renal capsule of the left kidney. Affi-5 and the appropriate isotype control were injected i.p. twice weekly at 10 or $20 \mathrm{mg} / \mathrm{kg}$, starting on day 2 after surgery. Tumor weight was determined at the end of the experiment by subtracting the right kidney weight from the weight of the tumorbearing left kidney.

Tumor growth was monitored after administration of luciferin ( $3 \mathrm{mg} /$ mouse, Sigma-Aldrich) with the IVIS Imaging System 100 (Xenogen Biosciences). Mice were sacrificed between days 17 and 12 after surgery.

The anti-MHCII blocking antibody (clone M5/114) and the isotype control (LTF-2) were obtained from BioXCell and administered i.p. at $10 \mathrm{mg} / \mathrm{kg} 1$ day prior to surgery and 3 times/week thereafter.

For the melanoma model, 8- to 12 -week-old C57BL/6 mice from Charles River Laboratories were injected subcutaneously with $1 \times 10^{5}$ $\mathrm{B} 16 \mathrm{FO}$ cells resuspended in PBS. Affi- 5 and the appropriate isotype control were injected i.p. twice weekly at $20 \mathrm{mg} / \mathrm{kg}$, starting on day 2 after surgery. Mice were sacrificed on day 18 after injection.

Flow cytometry and sorting. Human cell lines were dissociated using cell dissociation buffer (Invitrogen) and stained with antiCCR4 (R\&D Systems, FAB1567P) or IgG2B isotype control (R\&D Systems). After washing, cells were incubated with Alexa Fluor 594-anti-mouse antibody (Invitrogen). Intracellular staining was achieved using saponin permeabilization before antibodies against CCL17 and CCL22 were applied (R\&D Systems, IC364IP and IC3361P, respectively). RENCA murine cells were stained with Affi5 or isotype control. After washing, cells were incubated with Alexa Fluor 488-anti-human antibody (Invitrogen).

Tumor-bearing left kidneys were chopped and incubated in $2 \mathrm{mg} /$ $\mathrm{ml}$ collagenase V (Sigma-Aldrich), and $25 \mu \mathrm{g} / \mathrm{ml}$ DNAse (Roche), in HBSS (Sigma-Aldrich) for 45 minutes at $37^{\circ} \mathrm{C}, 5 \% \mathrm{CO}_{2}$. Tumor-bearing kidneys were selected to be representative of the average tumor weight of the treatment group they belonged to. The lysate was strained with $70-\mu \mathrm{m}$ strainers (Fisher Scientific), and red blood cells were lysed (BD Pharm Lyse, BD Biosciences). Cells were counted, and $6 \times 10^{6}$ cells were stained in PBS $+2 \%$ heat-inactivated FBS +2 mM EDTA after blocking with $\alpha$ CD16/CD32 (14-0161, eBioscience) for 15 minutes. Staining antibodies were diluted 1:200 unless otherwise specified: $\alpha$ CD45 (48-0451, eBioscience), $\alpha$ FoxP3 (56-5773, eBioscience), $\alpha$ CD3 (45-0031, eBioscience), $\alpha \mathrm{CD} 4$ (560783, BD Biosciences, 1:300), $\alpha \mathrm{CD} 8$ (48-0041, eBioscience, 1:300), $\alpha$ Ly6-C (45-5932, eBioscience), $\alpha$ Ly6-G (referred to here as Gr1) (35-5931, eBioscience), $\alpha \mathrm{CD} 49 \mathrm{~b}$ (108918, BioLegend), $\alpha \mathrm{F} 4 / 80$ 
(47-4801, eBioscience, 1:150), aCD11b (11-0112, eBioscience), aMMR (141706, BioLegend), $\alpha$ MHCII (17-5321, eBioscience), aCCR4 (131214, BioLegend), $\alpha \mathrm{CD} 4$ (560783, BD Biosciences, 1:300), $\alpha \mathrm{Ki}-67$ (612472, BD Biosciences, $5 \mu \mathrm{l} /$ staining). Appropriate isotype control antibodies were used to generate fluorescence minus one (FMO) controls. Viability was assessed with Fixable Viability Dye eFluor 780 or 506 (eBioscience) diluted 1:1,000. Staining was performed for 30 minutes at $4^{\circ} \mathrm{C}$. Cells were washed, fixed in $2 \%$ formaldehyde, and analyzed using a BD LSR Fortessa cytometer. Analysis was performed with FlowJo software.

For the sorting of TAMs, tumors were dissected and dissociated as described above. Cells were stained in PBS $+2 \%$ heat-inactivated FBS +2 mM EDTA after blocking with $\alpha$ CD16/CD32 (14-0161, eBioscience) for 15 minutes. Staining antibodies were from eBioscience and were diluted 1:200 unless otherwise specified: $\alpha$ CD45 (catalog 48-0451), $\alpha$ Ly6-G (Gr1) (catalog 35-5931), $\alpha$ F4/80 (catalog 47-4801, 1:150), $\alpha$ CD11b (cata$\log 11-0112)$, for 30 minutes at $4^{\circ} \mathrm{C}$. DAPI $(2.5 \mu \mathrm{g} / \mathrm{ml})$ was added prior to sorting, which was performed with a BD FACSAria II cell sorter.

Suppression assay. Tumors were dissociated as for flow cytometry staining and pooled. MDSCs were purified according to the manufacturer's instructions using the Myeloid-Derived Suppressor Cell Isolation kit (Miltenyi Biotec). Naive $\mathrm{CD}^{+}$cells were isolated from spleens of healthy mice using the Dynabeads FlowComp Pan T kit (Invitrogen). Before stimulation, $\mathrm{CD}^{+}$cells were pre-labeled with 5 $\mu \mathrm{M}$ CSFE (eBioscience) for 5 minutes at $37^{\circ} \mathrm{C}$ in medium and washed. Some of the cells were left without stimulus, while the remaining cells were stimulated with anti-CD3/anti-CD28-coated beads (Dynabeads Mouse T-Activator CD3/CD28, Invitrogen) at a ratio of 1:2 (beads/ CD3) and plated $\left(5 \times 10^{4} /\right.$ well $)$ in round-bottom 96-well plates. MDSCs were added to the wells at 10:1, 1:1, 1:2, and 1:8 ratios (CD3/ MDSCs) and incubated for 3 days. At the end of the incubation period, cells were collected and stained for viability, CD11b, CD3, CD4, and CD8 and analyzed by flow cytometry. Staining was performed for 30 minutes at $4^{\circ} \mathrm{C}$. Cells were washed, fixed in $2 \%$ formaldeyde, and analyzed using a BD LSR Fortessa cytometer. Analysis was performed with FlowJo software. $\mathrm{CD} 4^{+}$or $\mathrm{CD}^{+}$cells that had undergone at least one cycle of cell division were gated as proliferating.

Intracellular flow cytometry. Tumors were dissected and dissociated as described above. For macrophage staining, cells were plated $\left(0.5 \times 10^{6} / 500 \mu \mathrm{l}\right.$ in a 24 -well plate $)$ and incubated overnight with Brefeldin A (Sigma-Aldrich), $20 \mu \mathrm{g} / \mathrm{ml}$. The following day, cells were stained in PBS $+2 \%$ heat-inactivated FBS +2 mM EDTA after blocking with $\alpha C D 16 / C D 32,1: 200$ (14-0161, eBioscience) for 15 minutes. Staining antibodies were from eBioscience and were diluted 1:200 unless otherwise specified: $\alpha \mathrm{CD} 45$ (catalog 48-0451), $\alpha$ Ly6-G (Gr1) (catalog 35-5931), $\alpha \mathrm{F} 4 / 80$ (catalog 47-4801, 1:150) $\alpha \mathrm{CD} 11 \mathrm{~b}$ (catalog 11-0112), and Fixable Viability Dye eFluor 780 or 506 (eBioscience) diluted 1:1,000 for 30 minutes at $4^{\circ} \mathrm{C}$. After washing, cells were fixed in Intracellular Fixation Buffer (eBioscience) for 20 minutes at room temperature. Cells were further permeabilized and stained in permeabilization buffer with $\alpha \mathrm{TNF}$ (11-7321-81, eBioscience) 1:75 in $25 \mu \mathrm{l}$, or the appropriate isotype control, for 30 minutes at $4^{\circ} \mathrm{C}$. After washing, cells were analyzed using a BD LSR Fortessa cytometer. Analysis was performed with FlowJo software. For CD4/CD8 staining, lymphocytes were purified using Dynabeads FlowComp Mouse Pan T (Invitrogen) according to the instructions, plated in $500 \mu \mathrm{l}$ in a 24 -well plate, incubated with PMA (50 ng/ml, Sigma-Aldrich) and ionomycin $(1 \mu \mathrm{g} / \mathrm{ml}$, Sigma-Aldrich) for 1 hour, and further incubated in the presence of
Brefeldin A (20 $\mu \mathrm{g} / \mathrm{ml}$, Sigma-Aldrich) for 3 hours. At the end of the incubation, cells were washed; blocked as above; stained with $\alpha \mathrm{CD} 3$ (45-0031, 1:200), $\alpha \mathrm{CD} 8$ (48-0041, 1:300), $\alpha \mathrm{CD} 4$ (47-0041, 1:200), from eBioscience, and Fixable Viability Dye eFluor 506, 1:1,000; fixed in $2 \%$ formalin; and stored overnight at $4^{\circ} \mathrm{C}$. The day after, cells were permeabilized and stained with $\alpha$ IFN- $\gamma(17-7311,1: 100)$ and $\alpha$ TNF (117321-81, 1:75), or the appropriate isotype controls, from eBioscience, in $25 \mu \mathrm{l}$ in permeabilization buffer for 30 minutes at $4^{\circ} \mathrm{C}$, and further processed and analyzed as specified above.

Cytokine expression. Frozen tumors were lysed with a gentleMACS M tube (Miltenyi Biotec) in PBS with Complete Protease Inhibitors (Roche). Triton X-100 was added (1\%), and lysates were cleared by centrifugation and applied to Proteome Profiler Mouse Cytokine Array Panel A membranes (R\&D Systems). An equivalent amount of 200- $\mu$ g tumor lysate was incubated according to the manufacturer's instructions. Film exposures were quantified using NIH ImageJ software, subtracting a lane background.

In vitro Th1 polarization assay. Splenocytes from healthy BALB/c mice were obtained by mashing spleens through a $70-\mu \mathrm{m}$ strainer and lysis of red blood cells. $\mathrm{CD} 4^{+}$cells were purified with the Miltenyi Biotec $\mathrm{CD}^{+} \mathrm{T}$ cell purification kit according to the manufacturer's instructions. $\mathrm{CD} 4^{+}$cells were resuspended in Iscove's modified DMEM (10\%FBS, $50 \mu \mathrm{M} \beta$-mercaptoethanol, $8 \mathrm{mM}$ glutamine) and stimulated with 1:1 Dynabeads Mouse T-Activator CD3/CD28 (Invitrogen), mouse IL-2 $20 \mathrm{ng} / \mathrm{ml}$, and mouse IL-12 $5 \mathrm{ng} / \mathrm{ml}$ (from R\&D Systems). The day after, the indicated concentrations of CCL17 or CCL22 in combination with Affi-5 $(30 \mu \mathrm{g} / \mathrm{ml})$ were added to the plate. Experiments with Affi- 5 were performed in Corning Ultra-Low attachment plasticware (Corning Life Sciences). Cells were stimulated with restimulation cocktail plus transport inhibitor from eBioscience for 5 hours and stained for 30 minutes at $4^{\circ} \mathrm{C}$ with $\alpha \mathrm{CD} 16 / \mathrm{CD} 32$ (14-0161, eBioscience, 1:200), Fixable Viability Dye eFluor 450 1:1000, $\alpha C D 4$ (560783, BD Horizon CD4, BD Biosciences, 1:300) in $50 \mu$ l FACS buffer. After washing, cells were permeabilized and stained with $\alpha$ IFN- $\gamma$ (17-7311, eBioscience, 1:100) or isotype control. After washing, cells were analyzed using a BD LSR Fortessa cytometer.

Immunofluorescence. Renal carcinoma 786-O and RENCA cells were cultivated on a chamber slide (Nalge Nunc International) for 1-2 days. Cells were then fixed for 30 minutes with $4 \%$ formaldehyde and permeabilized with $0.5 \%$ Triton X-100 in PBS for 10 minutes. Samples were blocked with $1 \%$ BSA for 2 hours at room temperature, then incubated overnight at $4^{\circ} \mathrm{C}$ with $25 \mathrm{mg} / \mathrm{ml} \alpha C C R 4$ (IMG-322, IMGENEX, Novus Biologicals). After washing, samples were incubated with Alexa Fluor 594-conjugated secondary antibody, 1:2,000, for 2 hours at room temperature. Finally, samples were washed and mounted with ProLong Gold DAPI (Invitrogen, P36931). Cells were then visualized using a Zeiss LSM510 confocal microscope.

RNA interference for CCR4 in renal cancer cell lines. Commercially available shRNAs, based on the pRS vector, were purchased from Origene Technologies. Four non-overlapping sequences were provided to target human (TR314127) and mouse (TR500386) CCR4. A nonspecific shRNA sequence (shGFP, TR30003) and empty pRS vector (TR20003) served as controls. Phoenix packaging cell line was transfected overnight with LipofectAMINE 2000 (Invitrogen) and $5 \mu \mathrm{g}$ of the shRNA plasmid DNA. After an incubation with complete medium at $33^{\circ} \mathrm{C}$ and $5 \% \mathrm{CO}_{2}$ for 16 hours, the supernatant was collected, filtered, and diluted 8:10 in RCC medium with the addition of Polybrene. 
RENCA or $786-O$ cells at $30 \%$ confluence were infected for 10 hours twice within 48 hours. Virus-infected cells were selected with $1.5 \mu \mathrm{g} / \mathrm{ml}$ puromycin (InvivoGen). For long-term silencing in vivo, RENCA-luc cells were infected with 3 lentiviral GIPZ vectors targeting mouse CCR4 from Thermo Fisher Scientific. Viral particles were obtained by transfecting HEK293T cells with the calcium phosphate method with $21 \mu \mathrm{g}$ shRNA construct, $7 \mu \mathrm{g}$ VSVG construct, $14 \mu \mathrm{g}$ HIV construct (from Addgene), in a 14-cm dish, and collecting the supernatant for 24 hours. RENCA-luc cells were infected for 24 hours in a 6-well plate with $1 \mathrm{ml}$ supernatant. Virus-infected cells were selected with $0.3 \mu \mathrm{g} / \mathrm{ml}$ puromycin (Sigma-Aldrich). After verifying the silencing, cells infected with vector V3LMM_439088 were chosen for the in vivo experiment.

Statistics. All data are expressed as mean \pm SEM. Differences were considered significant at $P<0.05$, using a Student's $t$ test (2-tailed), ANOVA test, or nonparametric test as appropriate, performed with the statistical analysis software Prism (GraphPad Software). $P$ values are specified.

Study approval. Patient samples were collected under the Multicentre Research Ethics Committee (MREC); Ethical Number MREC 02/8/78. All patients provided written informed consent. All experimental procedures were performed according to a protocol approved under Home Office license 70/7411 and according to the Animal Welfare and Ethical Review Body (AWERB) of Queen Mary University of London.

\section{Author contributions}

$\mathrm{CB}$ designed, performed, and interpreted most of the experiments, and wrote the manuscript. MNK designed, performed, and interpreted the experiments shown in Figure 1 and Supplemental Figures 1-3. TS, AM, and EM contributed to designing, performing, and interpreting some experiments and editing the manuscript. RT performed most of the surgery experiments. MJ conducted and analyzed the B16 melanoma studies. MC measured and analyzed the chemokine levels in patients. HK contributed to the design of the research studies. UBH and ARD provided the Affi- 5 antibody and contributed to the design of the experiments. LF and RWW contributed to the design of the research studies and editing the manuscript. TP provided patient samples, patient information, and intellectual input. SAQ contributed to designing and interpreting experiments analyzing adaptive immunity. FRB conceived of, designed, and supervised the project and experimental plan, interpreted experiments, and wrote the manuscript.

\section{Acknowledgments}

The study was supported by Cancer Research UK (CRUK) programme grant C587/A16354 and a research grant from Affitech AS. The authors would like to thank Yaohe Wang (Barts Cancer Institute) for help in setting up CCR4, CCL17, and CCL22 IHC staining; David Gould (William Harvey Research Institute) for luciferase-labeling renal cancer cell lines; Melania Capasso and Mark Brown (Barts Cancer Institute) for assistance with the silencing experiments; Alzbeta Talarovicova (Barts Cancer Institute) for assistance with the mouse work; and Matthew McCourt (Actigen) for helpful discussion.

Address correspondence to: Frances R. Balkwill, Barts Cancer Institute, Queen Mary University of London, Charterhouse Square, London EC1M 6BQ, United Kingdom. Phone: 442078823576; E-mail: f.balkwill@qmul.ac.uk.
1. Raman D, Sobolik-Delmaire T, Richmond A. Chemokines in health and disease. Exp Cell Res. 2011;317(5):575-589.

2. Allavena P, Germano G, Marchesi F, Mantovani A. Chemokines in cancer related inflammation. Exp Cell Res. 2011;317(5):664-673.

3. Mantovani A, Savino B, Locati M, Zammataro L, Allavena P, Bonecchi R. The chemokine system in cancer biology and therapy. Cytokine Growth Factor Rev. 2010;21(1):27-39.

4. Zlotnik A, Burkhardt AM, Homey B. Homeostatic chemokine receptors and organ-specific metastasis. Nat Rev Immunol. 2011;11(9):597-606.

5. Balkwill FR. The chemokine system and cancer. JPathol. 2012;226(2):148-157.

6. Bonecchi R, et al. Differential expression of chemokine receptors and chemotactic responsiveness of type $1 \mathrm{~T}$ helper cells (Th1s) and Th2s. J Exp Med.1998;187(1):129-134.

7. Kunkel EJ, et al. Expression of the chemokine receptors CCR4, CCR5, and CXCR3 by human tissue-infiltrating lymphocytes. Am J Pathol. 2002;160(1):347-355.

8. Imai T, et al. Selective recruitment of CCR4bearing Th 2 cells toward antigen-presenting cells by the CC chemokines thymus and activationregulated chemokine and macrophagederived chemokine. Int Immunol. 1999;11(1):81-88.

9. Yoshie O, Matsushima K. CCR4 and its ligands: from bench to bedside. Int Immunol. 2015;27(1):11-20.

10. Curiel TJ, et al. Specific recruitment of regulatory
T cells in ovarian carcinoma fosters immune privilege and predicts reduced survival. Nat Med. 2004;10(9):942-949.

11. Jacobs JF, et al. Prognostic significance and mechanism of Treg infiltration in human brain tumors. J Neuroimmunol. 2010;225(1-2):195-199.

12. Yang P, et al. TGF- $\beta$-miR-34a-CCL22 signaling-induced Treg cell recruitment promotes venous metastases of HBV-positive hepatocellular carcinoma. Cancer Cell. 2012;22(3):291-303.

13. Gobert M, et al. Regulatory $\mathrm{T}$ cells recruited through CCL22/CCR4 are selectively activated in lymphoid infiltrates surrounding primary breast tumors and lead to an adverse clinical outcome. Cancer Res. 2009;69(5):2000-2009.

14. Li JY, et al. The chemokine receptor CCR4 promotes tumor growth and lung metastasis in breast cancer. Breast Cancer Res Treat. 2012;131(3):837-848.

15. Lee JH, et al. The chemokine receptor CCR4 is expressed and associated with a poor prognosis in patients with gastric cancer. Ann Surg. 2009;249(6):933-941.

16. Ishida T, et al. Defucosylated anti-CCR4 monoclonal antibody (KW-0761) for relapsed adult T-cell leukemia-lymphoma: a multicenter phase II study. J Clin Oncol. 2012;30(8):837-842.

17. Ogura M, et al. Multicenter phase II study of mogamulizumab (KW-0761), a defucosylated anti-cc chemokine receptor 4 antibody, in patients with relapsed peripheral T-cell lymphoma and cutaneous T-cell lymphoma. JClin Oncol.
2014;32(11):1157-1163.

18. Sugiyama D, et al. Anti-CCR4 mAb selectively depletes effector-type FoxP3+CD4+ regulatory $\mathrm{T}$ cells, evoking antitumor immune responses in humans. Proc Natl Acad Sci USA. 2013;110(44):17945-17950.

19. Hagemann UB, et al. Fully human antagonistic antibodies against CCR4 potently inhibit cell signaling and chemotaxis. PLoS ONE. 2014;9(7):e103776.

20. Sica A, et al. Macrophage polarization in tumour progression. Semin Cancer Biol. 2008;18(5):349-355.

21. Porta C, et al. Tolerance and M2 (alternative) macrophage polarization are related processes orchestrated by 50 nuclear factor kappaB. Proc Natl Acad Sci USA. 2009;106(35):14978-14983.

22. Overdijk MB, et al. Crosstalk between human IgG isotypes and murine effector cells. Jimmunol. 2012;189(7):3430-3438.

23. Liu Q, et al. Expression of chemokine receptor 4 was associated with poor survival in renal cell carcinoma. Med Oncol. 2014;31(4):882.

24. Pyonteck SM, et al. CSF-1R inhibition alters macrophage polarization and blocks glioma progression. Nat Med. 2013;19(10):1264-1272.

25. Dannenmann SR, et al. Tumor-associated macrophages subvert $\mathrm{T}$-cell function and correlate with reduced survival in clear cell renal cell carcinoma. Oncoimmunology. 2013;2(3):e23562.

26. Beck A, Reichert JM. Marketing approval of mogamulizumab: a triumph for glyco-engineering. MAbs. 2012;4(4):419-425. 